\title{
Decision Sciences, Economics, Finance, Business, Computing, and Big Data: Connections
}

Instituto

Complutense de Análisis Económico

\section{Chia-Lin Chang}

Department of Applied Economics and Department of Finance

National Chung Hsing University, Taiwan

\section{Michael McAleer}

Department of Finance, Asia University, Taiwan and

Discipline of Business Analytics, University of Sydney Business School, Australia

And Econometric Institute, Erasmus School of Economics,

Erasmus University Rotterdam, The Netherlands and

Department of Economic Analysis and ICAE, Complutense University of Madrid, Spain and Institute of Advanced Sciences

Yokohama National University, Japan

\section{Wing-Keung Wong}

Department of Finance, Fintech Center, and Big Data Research Center, Asia University,

Taiwan and Department of Medical Research

China Medical University Hospital And Department of Economics and Finance

Hang Seng Management College, Hong Kong, China and Department of Economics, Lingnan University, Hong Kong, China

\begin{abstract}
This paper provides a review of some connecting literature in Decision Sciences, Economics, Finance, Business, Computing, and Big Data. We then discuss some research that is related to the six cognate disciplines. Academics could develop theoretical models and subsequent econometric and statistical models to estimate the parameters in the associated models. Moreover, they could then conduct simulations to examine whether the estimators or statistics in the new theories on estimation and hypothesis have small size and high power. Thereafter, academics and practitioners could then apply their theories to analyze interesting problems and issues in the six disciplines and other cognate areas.
\end{abstract}

Keywords Decision sciences, economics, finance, business, computing, and big data, theoretical models, econometric and statistical models, applications.
JEL Classification
A10, G00, G31, O32.

\section{Working Paper no 1809 \\ March, 2018}




\title{
Decision Sciences, Economics, Finance, Business, Computing, and Big Data: Connections
}

\author{
Chia-Lin Chang \\ Department of Applied Economics and Department of Finance \\ National Chung Hsing University, Taiwan \\ Michael McAleer \\ Department of Finance, Asia University, Taiwan \\ and \\ Discipline of Business Analytics, University of Sydney Business School, Australia \\ and \\ Econometric Institute, Erasmus School of Economics, \\ Erasmus University Rotterdam, The Netherlands \\ and \\ Department of Economic Analysis and ICAE, Complutense University of Madrid, Spain \\ and \\ Institute of Advanced Sciences, Yokohama National University, Japan

\section{Wing-Keung Wong} \\ Department of Finance, Fintech Center, and Big Data Research Center, Asia University, Taiwan \\ and \\ Department of Medical Research, China Medical University Hospital \\ and \\ Department of Economics and Finance, Hang Seng Management College, Hong Kong, China \\ and \\ Department of Economics, Lingnan University, Hong Kong, China
}

March 2018

* For financial and research support, the first author is grateful to the National Science Council, Ministry of Science and Technology (MOST), Taiwan, the second author wishes to thank the Australian Research Council and National Science Council, Ministry of Science and Technology (MOST), Taiwan, and the third author acknowledges the Research Grants Council of Hong Kong, Asia University, China Medical University Hospital, Hang Seng Management College, Lingnan University, and National Science Council, Ministry of Science and Technology (MOST), Taiwan The third author would also like to thank Robert B. Miller and Howard E. Thompson for their continuous guidance and encouragement. 


\begin{abstract}
This paper provides a review of some connecting literature in Decision Sciences, Economics, Finance, Business, Computing, and Big Data. We then discuss some research that is related to the six cognate disciplines. Academics could develop theoretical models and subsequent econometric and statistical models to estimate the parameters in the associated models. Moreover, they could then conduct simulations to examine whether the estimators or statistics in the new theories on estimation and hypothesis have small size and high power. Thereafter, academics and practitioners could then apply their theories to analyze interesting problems and issues in the six disciplines and other cognate areas.
\end{abstract}

Keywords: Decision sciences, economics, finance, business, computing, and big data, theoretical models, econometric and statistical models, applications.

JEL: A10, G00, G31, O32. 


\section{Introduction}

There are many studies that link Decision Sciences, Economics, Finance, Business, Computing, and Big Data. In addition, Decision Sciences is related to many different though cognate areas, including Science, Engineering, Medical Science, Experimental Science, Psychology, Social Science, Political Science, Management, and Business.

In this paper, we will discuss different types of utility functions, stochastic dominance (SD), mean-risk (MR) models, portfolio optimization (PO), and other financial, economic, business, marketing, and management models that can be used in computational science and Big Data. Academics could develop theoretical models and thereafter develop econometric and statistical models to estimate the associated parameters to analyze a number of interesting issues in Decision Sciences, Economics, Finance, Business, Computing, and Big Data. Academics could then conduct simulations to examine whether the estimators or statistics in the theories on estimation and hypothesis develop have small size and high power. Thereafter, academics and practitioners could apply the novel and innovative theories to analyze a number of interesting issues in the six disciplines and cognate areas.

The plan of the remainder of the paper is as follows. In Section 2, some theoretical models are dissected and discussed. Alternative statistical and econometrics models are analyzed critically in Section 3. Alternative procedures for conducting simulations are discussed in Section 4. A brief discussion of empirical models is presented in Section 5, followed by some concluding remarks in Section 6.

\section{Theoretical Models}

It is important to commence any rigorous research in Decision Sciences, Economics, Finance, Business, Computing, and Big Data by developing appropriate theoretical models. The recent literature in these disciplines has been concerned with developing theories to extend those that have been discussed in a number of existing reviews. We discuss some of our recent research in the following subsections. 


\subsection{Utility Functions}

Before the $17^{\text {th }}$ Century, most scholars considered investors to be risk neutral because they valued a gamble at its expected value. Bernoulli (1738) argues that investors were risk averse. In reality, individuals are not always risk averse or risk neutral. Many individuals engage in regular risk-seeking activity, such as buying lottery tickets. Hammond (1974), Stoyan (1983), Wong and Li (1999), Li and Wong (1999), Wong (2007), Guo and Wong (2016), among others, argue that investors could be either riskaverse or risk-seeking.

Examining the relative attractiveness of various forms of investments, Friedman and Savage (1948) claim that the strictly concave functions may not be able to explain the behavior why investors buy insurance or lottery tickets. Markowitz (1952a) was the first to address Friedman and Savage's concern, by proposing a utility function which could have convex and concave regions in both the positive and negative domains. Kahneman and Tversky (1979) identify circumstances in which individuals behave in a risk-seeking manner by allowing investors to follow S-shaped "utility” functions that they referred to as "value functions”.

Markowitz (1952), Levy and Levy (2002, 2004), Wong and Chan (2008), among others, suggest investors could follow S-shaped as well as reverse S-shaped utility functions. Broll, Egozcue, Wong, Zitikis (2010), and Egozcue, Fuentes García, Wong, and Zitikis (2011) argue that investment behavior for investors could follow S-shaped as well as reverse S-shaped utility functions. Recently, Guo, Qiao, and Wong (2017) introduce a new utility function for investors that consists of both risk-averse and risk-seeking components to provide a new solution to answer the observation posed by Friedman and Savage (1948) that investors buy insurance and also try their luck with lotteries.

Egozcue, Guo, and Wong (2015), among others, develop theory for the competitive firm with regret-averse utility functions, while Guo, Egozcue, and Wong (2017) develop an optimal production decision theory for firms with disappointment-aversion utility functions. 
Lien (2008) compares the exponential utility function with its second-order approximation under the normality distribution assumption in the optimal production and hedging decision framework. Guo, Lien, and Wong (2016) extend the theory by comparing the exponential utility function with a $2 n$-order approximation for any integer $n$. The authors also propose an approach with an illustration to find the smallest $n$ that provides a good approximation.

We have been developing some theories, estimation, and testing to analyse different utility functions and the preferences of different types of investors. We summarize some of the results below, and refer to Sriboonchitta, Wong, Dhompongsa, and Nguyen (2009) and Bai, Guo, Li, and Wong (2017) for further information.

\subsection{Stochastic Dominance}

Several researchers have been developing a range of interesting theories in stochastic dominance, and these will be discussed in the following sections.

\subsubsection{Stochastic Dominance for Risk Averters and Risk Seekers}

Wong and Li (1999) develop several properties for convex stochastic dominance to compare the preferences of different combinations of several assets for both risk-averse and risk-seeking investors. In addition, they compare the preferences between a convex combinations of several continuous distributions and a single continuous distribution. In addition, Li and Wong (1999) develop several SD theorems for the location-and-scale family, and linear combinations of random variables for risk seekers, as well as risk averters.

Wong (2007) extends their work by introducing the first three orders of both ascending SD (ASD) and descending SD (DSD) to decisions in business planning and investment to risk-averse

and risk-seeking decision makers, so that they can compare both returns and losses. They provide tools to identify the first-order SD prospects and discern arbitrage opportunities that could increase their expected utility and expected wealth. They also introduce the mean-variance (MV) rule to decisions in business planning or investment on both returns and losses for both risk- 
averse and risk-seeking decision makers, and show that the rule is equivalent to the SD rule under certain regularity conditions.

Chan, Clark, and Wong (2016) analyze properties of SD for both risk-averse and risk-seeking SD (RSD) for risk-seeking investors which, in turn, enables an examination of their behavior. The authors first discuss the basic property of SD and RSD that links the SD and RSD to expectedutility maximization. Thereafter, they prove that an hierarchy exists in both SD and RSD relationships, and that the higher orders of SD and RSD can be inferred by the lower orders of SD and RSD, but not vice-versa. Furthermore, they examine the conditions in which third-order SD preferences are 'the opposite of' or 'the same as' their counterpart third-order RSD preferences. In addition, they establish the relationship between the order of the variances and that of the integrals for two assets, which enables the establishment of certain relationships between the dominance of the variances and the second- and third-order SD and RSD for two assets under the condition of equal means.

The theory developed in their paper provides a set of tools that enables investors to identify prospects for first-, second-, and third-order SD and RSD, and thereby enables investors to improve their investment decisions. Another contribution of the paper is that the authors recommend checking the dominance of the means of the distributions to draw inferences for the preferences for two different assets for third-order risk averters and risk seekers. They illustrate this idea by comparing the investment behavior of both third-order risk averters and risk seekers in bonds and stocks.

Guo and Wong (2016) extend some univariate SD results to multivariate SD (MSD) for both risk averters and risk seekers, respectively, to $n$ order for any $n>0$ when the attributes are assumed to be independent, and utility is assumed to be additively separable. Under these assumptions, they develop several properties for MSD for both risk averters and risk seekers. For example, they prove that MSD is equivalent to expected-utility maximization for both risk averters and risk seekers, respectively. 
The authors show that the hierarchical relationship exists for MSD, and establish several dual relationships between the MSD for risk averters and risk seekers. They develop properties for non-negative combinations and convex combinations of random variables of MSD, and develop the theory of MSD for the preferences of both risk averters and risk seekers on diversification. In addition, they discuss some MSD relationships when the attributes are dependent and discuss the importance and use of the novel theoretical results developed in the paper.

\subsubsection{Stochastic Dominance for Investors with S-shaped and Reverse S-shaped Utility Functions}

Wong and Chan (2008) extend the work on Prospect SD (PSD) and Markowitz SD (MSD) to the first three orders, and link the corresponding S-shaped and reverse S-shaped utility functions to the first three orders. They conducted experiments to illustrate each case of MSD and PSD to the first three orders, and demonstrate that the higher-order MSD and PSD cannot be replaced by lower-order MSD and PSD. Furthermore, they show that an hierarchy exists in both PSD and MSD relationships, arbitrage opportunities exist in the first orders of both PSD and MSD, and for any two prospects under certain conditions, the third-order MSD preference will be "the opposite of" or "the same as" their counterpart third-order PSD preferences.

\subsubsection{Stochastic Dominance Theory for Investors with Both Risk-Averse and Risk- Seeking Components}

Recently, Guo, Qiao, and Wong (2017) develop a new SD theory for investors with AD utility that consists of both risk-averse and risk-seeking components. Based on the theory, they not only find a new solution to answer the observation posed by Friedman and Savage (1948) that people could buy insurance and also try their luck with lotteries. They also find that investors with AD utility will buy both bonds and stocks, as well as both bonds and futures, to achieve higher expected utility. The authors also developed several properties, including expected-utility maximization, hierarchy, transitivity, and diversification, for the new SD theory for investors with AD utility.

\subsubsection{Almost Stochastic Dominance}


Guo, Zhu, Wong, and Zhu (2013) provide further information on both expected-utility maximization and the hierarchy property. For almost SD (ASD), Leshno and Levy (2002) propose a definition, while Tzeng et al. (2013) modify it, to provide a further separate definition. Interestingly, Guo, Zhu, Wong, and Zhu (2013) show that the former has the hierarchy property but not expected utility maximization, whereas the latter has expected utility maximization but not the hierarchy property.

Guo, Post, Wong, and Zhu, (2014) establish necessary conditions for ASD criteria of various orders. These conditions take the form of restrictions on algebraic combinations of moments of the probability distributions under consideration. The relevant set of conditions depends on the relevant order of ASD, but not on the critical value for the admissible violation area. These conditions can help to reduce the information requirement and computational burden in practical applications. A numerical example and an empirical application to historical stock market data illustrate the moment conditions. The first four moment conditions, in particular, seem appealing for many applications.

Guo, Wong, and Zhu (2016) extend ASD theory for risk averters to include ASD for risk-seeking investors. Thereafter, they examine the relationship between ASD for risk seekers, on the one hand, and ASD for risk averters, on the other. Tsetlin, et al. (2015) develop the theory of generalized ASD (GASD). Guo, Wong, and Zhu (2016) discuss the advantages and disadvantages of ASD and GASD.

\subsection{Portfolio Optimization}

The mean-variance (MV) portfolio optimization procedure is the milestone of modern finance theory for asset allocation, investment diversification, and optimal portfolio construction (Markowitz, 1952b). In the procedure, investors select portfolios that maximize profit subject to

achieving a specified level of calculated risk or, equivalently, minimize variance subject to obtaining a predetermined level of expected gain. 
However, the estimates have been demonstrated to depart seriously from the theoretical optimal return. Michaud (1989) and others have found the MV-optimized portfolios do more harm than good, while Bai et al. (2009a) have proved that this phenomenon is natural.

Recently, Li, Li, Hui, and Wong (2017) extend Maslow's (1943) need hierarchy theory and the two-level optimization approach by developing the framework of the Malsow portfolio selection model (MPSM). The authors solve two optimization problems to meet the needs of individuals with low financial sustainability who prefer to satisfy their lower-level (safety) needs first, and thereafter seek higher-level (self-actualization) needs to maximize the optimal returns.

\subsection{Risk Measures}

Several researchers have been developing properties for several risk measures to be used in finance, economics, and related cognate disciplines. We briefly discuss the properties for some risk measures in the following sub-sections.

\subsubsection{Sharpe Ratio}

The theory of modern finance starts from the mean-variance portfolio optimization developed by Markowitz (1952b). The Sharpe ratio (Sharpe 1966), the ratio of the excess expected return of an investment to its return volatility or standard deviation, is one of the most commonly-used measures in the financial mean-variance framework.

\subsubsection{VaR and CVaR}

Ma and Wong (2010) establish some behavioral foundations for various types of Value-at-Risk (VaR) models, including VaR and conditional VaR (CVaR), as measures of downside risk. They establish some logical connections among VaRs, VaRs, SD, and utility maximization. Though they are supported to a certain extent with unanimous choices by some specific groups of expected or non-expected-utility investors, VaRs as profiles of risk measures at various levels of 
risk tolerance are not quantifiable. This arises as they can only provide partial and incomplete risk assessments for risky prospects.

The authors also include in the discussion the relevant VaRs and several alternative risk measures for investors. These alternatives use somewhat weaker assumptions about risk-averse behavior by incorporating a mean-preserving spread. For this latter group of investors, the authors provide arguments for and against the standard deviation versus VaR and CVaR as objective and quantifiable measures of risk in the context of portfolio choice.

\subsubsection{Omega Ratio}

It is well-known (Wong, 2007) that, under certain conditions, the mean-variance rule is equivalent to the stochastic dominance rule. Some academics hypothesize that there could exist a mean-Omega ratio rule that could be equivalent to the stochastic dominance rule under certain regularity conditions.

Both SD and Omega ratio can be used to examine whether the market is efficient, if there is any arbitrage opportunity in the market, and if there might be any anomaly in the market. Guo, Jiang, and Wong (2017) evaluate the relationship between SD and the Omega ratio. They find that second-order SD and/or second-order risk-seeking SD (RSD) alone for any two prospects is not sufficient to imply Omega ratio dominance, as the Omega ratio of one asset is always greater than that of the other. They extend the theory of risk measures by proving that the preference of second-order SD implies the preference of the corresponding Omega ratio only when the return threshold is less than the mean of the higher returns asset.

On the other hand, the preference of the second-order RSD implies the preference of the corresponding Omega ratio only when the return threshold is larger than the mean of the smaller returns asset. Nonetheless, it is shown that first-order SD implies Omega ratio dominance. Thereafter, the authors apply the theory to examine the relationship between property size and

property investment in the Hong Kong real estate market. The authors conclude that the Hong 
Kong real estate market is not efficient and there are expected arbitrage opportunities and anomalies in the Hong Kong market.

\subsubsection{First- and High-order Risk Measures}

Farinelli and Tibiletti (2008), hereafter F-T, propose a general risk-reward performance measurement ratio. Due to its simplicity and generality, the F-T ratios have gained much attention. F-T ratios are functions of average gains to average losses with respect to a target, each raised by some power index. Omega ratio and Upside Potential ratio are both special cases of FT ratios. Niu, Wong, and Zhu (2016) establish the consistency of F-T ratios with respect to firstorder stochastic dominance. It is shown that second-order stochastic dominance is not consistent with the F-T ratios. This outcome is illustrated by a simple example. Niu, Wong, and Xu (2017) further extend the theory and show the sufficient relationship between the $(n+1)$-order SD and the $n$-order Kappa ratio.

The authors clarify the restrictions on necessarily beating the target for the higher-order SD consistency of the Kappa ratios. Thereafter, they show that, in general, the necessary relationship between SD/RSD and the Kappa ratio cannot be established. They find that, when the variables being compared belong to the same location-scale family, or the same linear combination of location-scale families, they can obtain the necessary relationship between the $(n+1)$-order SD with the $n$-order Kappa ratio after imposing certain conditions on the means.

\subsection{Cost of Capital}

Gordon and Shapiro (1956) develop the dividend yield plus growth model for individual firms, while Thompson (1985) improves the theory by combining the model with an analysis of past dividends to estimate the cost of capital and its 'reliability'. Thompson and Wong (1991) estimate the cost of capital using discounted cash flow (DCF) methods that require forecasting dividends. Thompson and Wong (1996) extend the theory by proving the existence and uniqueness of a solution for the cost of equity capital, and the cost of equity function is 
continuously differentiable. Wong and Chan (2004) extend their theory further by proving the existence and uniqueness of the reliability.

\subsection{Behavorial Models}

Barberis, Shleifer and Vishny (1998), among others, use Bayesian models to explain investors' behavioral biases by incorporating the conservatism heuristics and representativeness heuristics in making decisions. Lam, Liu, and Wong (2010) extend the theory by developing a model of weight assignments using a pseudo-Bayesian approach that reflects investors' behavioral biases. They use the model to explain several financial anomalies, including excess, volatility, short-run underreaction, long-run overreaction, and magnitude effects. Lam, Liu, and Wong (2012) extend their work further by developing additional properties for the pseudo-Bayesian approach that reflects investors' behavioral biases, and by explaining the linkages between these market anomalies and investors' behavioral biases.

Fung, Lam, Siu, and Wong (1998) extend the theory by incorporating the pseudo-Bayesian model with the impact of a financial crisis. They derive several properties of stock returns during the financial crisis, in addition to recovery from the crisis. Guo, McAleer, Wong, and Zhu (2017) extend the model by assuming that the earnings shock of an asset follows a random walk model, with and without drift, to incorporate the impact of financial crises. The authors assume that the earning shock follows an exponential family distribution to accommodate symmetric as well as asymmetric information.

By using this model setting, they develop several properties on the expected earnings shock and its volatility, and establish properties of investor behavior on the stock price and its volatility, during financial crises and the subsequent recovery. Thereafter, they develop properties to explain excess volatility, short-term underreaction, long-term overreaction, and their magnitude effects during financial crises and the subsequent recovery. .

Egozcue and Wong (2010a) extend prospect theory, mental accounting, and the hedonic editing model by developing an analytical theory that explains the behavior of investors with extended 
value functions, in segregating or integrating multiple outcomes in the process of evaluating mental accounting.

Whether to keep products segregated (for example, unbundled) or integrate some or all of them (for example, bundled) has been a problem of profound interest to theorists and practitioners in areas such as portfolio theory in finance, risk capital allocations in insurance, and marketing of consumer products. Such decisions are inherently complex, and depend on factors such as the underlying product values and consumer preferences, the latter being frequently described using value functions, which are also known as utility functions in economics. Egozcue, Fuentes García, Wong, and Zitikis (2012a) develop decision rules for multiple products, which are generally referred to as 'exposure units' to cover naturally manifold scenarios spanning well beyond 'products'.

The findings show, for example, that the celebrated Thaler's principles of mental accounting hold as originally postulated when the values of all exposure units are positive (that is, all are gains) or all negative (that is, all are losses). In the case of exposure units with mixed-sign values, decision rules are much more complex and rely on cataloging the Bell-number of cases that grow very fast, which also depends on the number of exposure units. Consequently, in the paper the authors provide detailed rules for the integration and segregation decisions for up to three exposure units, and partial rules for an arbitrary number of units

It should be noted that the theory of decision making behavior developed by Egozcue and Wong (2010a) and Egozcue, Fuentes García, Wong, and Zitikis (2012a) is for the marketing discipline, and they develop a theory of consumer behavior.

\subsection{Indifference Curves}

Meyer (1987) extends MV theory to include the comparison among distributions that differ only by location and scale parameters, and to include general utility functions with only convexity or concavity restrictions. Wong (2006) extends the theoretical results of both Meyer (1987) and Tobin (1958) by showing that the indifference curve is convex upwards for risk averters, 
concave downwards for risk lovers, and horizontal for risk neutral investors, to include the general conditions stated by Meyer (1987). In addition, Wong (2006) develops several properties among the first- and second-order SD efficient sets and the mean-variance efficient set.

Wong and Ma (2008) extend the work on the location-scale (LS) family with general $n$ random seed sources in the multivariate setting. In addition, the authors develop several properties for certain general non-expected utility functions that are defined over the LS family, and characterize the shapes of the indifference curves that are induced by the location-scale expected utility functions and non-expected utility functions. Thereafter, the authors develop some properties of several well-defined partial orders and dominance relations that are defined over the LS family, including first- and second-order stochastic dominance, the mean-variance rule, and location-scale dominance.

Broll, Egozcue, Wong, and Zitikis (2010) discuss prospect theory and establish general results concerning certain covariances from which they can, in turn, infer the properties of indifference curves and hedging decisions within prospect theory.

\subsection{Diversification}

Wong and $\mathrm{Li}$ (1999) extend the theory of convex SD (Fishburn, 1974) by including any distribution function, developing the results for both risk seekers and risk averters, and including third-order stochastic dominance. Their results can be used to extend a theorem of Bawa et al. (1985) on comparisons between a convex combinations of several continuous distributions and a single continuous distribution.

$\mathrm{Li}$ and Wong (1999) develop some results for diversification preferences for risk averters and risk seekers. Egozcue and Wong (2010) incorporate both majorization theory and SD theory to develop a general theory. The authors provide a unifying framework for determining the diversification preferences of risk-averse investors and conditions under which they would unanimously judge a particular asset to be superior. In particular, they develop a theory for 
comparing the preferences of different convex combinations of assets that characterize a portfolio to yield higher expected utility by using second-order SD.

Egozcue, Fuentes García, Wong, and Zitikis (2011) evaluate rankings of completely and partially diversified portfolios, and also of specialized assets, when investors follow so-called Markowitz preferences. It can be shown that diversification strategies for Markowitz investors are more complex than in the case of risk-averse and risk-inclined investors, whose investment strategies have been investigated extensively in the literature. In particular, the authors observe that, for Markowitz investors, preferences toward risk vary depending on their sensitivities toward gains and losses.

For example, it can be shown that, unlike the case of risk-averse and risk-inclined investors, Markowitz investors might prefer investing their entire wealth in just a single asset. This interesting result enables a better understanding of some financial anomalies and puzzles, such as the well-known diversification puzzle, which notes that some investors tend to concentrate on investing in only a few assets instead of choosing the seemingly more attractive complete diversification approach.

Lozza, Wong, Fabozzi, Egozcue (2017) provide a general valuation of the diversification attitude of investors. First, they empirically examine the diversification of mean-variance optimal choices in the US stock market during the 11-year period 2003-2013. Then they analyze the diversification problem from the perspective of risk-averse investors and risk-seeking investors. Second, the authors prove that investors' optimal choices will be similar if their utility functions are not too distant, independent of their tolerance (or aversion) to risk. Finally, they discuss investors' attitudes towards diversification when the choices available to investors depend on several parameters.

\subsection{Two-Moment Decision Model}

Alghalith, Guo, Niu, and Wong (2017) analyze the impacts of joint energy and output prices uncertainties on the input demands in a mean-variance framework. They find that an increase in 
the expected output price will surely cause the risk-averse firm to increase the input demand, while an increase in the expected energy price will surely cause the risk-averse firm to decrease the demand for energy, but increase the demand for the non-risky inputs. Furthermore, the authors investigate the two cases with only uncertain energy price and only uncertain output price, respectively. In the case with only uncertain energy price, they find that the uncertain energy price has no impact on the demand for the non-risky inputs. They also show that the concepts of elasticity and decreasing absolute risk aversion (DARA) play an important role in the comparative statics analysis.

Alghalith, Niu, and Wong (2017) analyze the impacts of joint energy and output prices uncertainties on the inputs demand in a mean-variance framework. They find that the concepts of elasticities and variance vulnerability play important roles in the comparative statics analysis. If the firms' preferences exhibit variance vulnerability, increasing the variance of energy prices will necessarily cause the risk averse firm to decrease the demand for the non-risky inputs. Furthermore, the authors investigate two special cases, namely with only uncertain energy price, on the one hand, and only uncertain output price, on the other. In the case with only uncertain energy price, it is found that the uncertain energy price has no impact on the demand for the nonrisky inputs. If the firms' preferences exhibit variance vulnerability, increasing the variance of energy prices will surely cause the risk averse firm to decrease the demand for energy.

With multiple additive risks, the mean-variance approach and the expected utility approach of risk preferences are compatible if all attainable distributions belong to the same location-scale family. Under this proviso, Guo, Wagener, Wong, and Zhu (2017) survey the existing results on the parallels of the two approaches with respect to risk attitudes, the changes thereof, and the comparative statics for simple linear choice problems under risk. In the mean-variance approach, all the effects can be couched in terms of the marginal rate of substitution between the mean and variance. The authors apply the theory stated and developed in the paper to evaluate the behavior of banking firms, and examine risk-taking behavior with background risk in the mean-variance model.

\subsection{Dynamic Models with Background Risk}


Alghalith, Guo, Wong, and Zhu (2016) use a general utility function to present two dynamic models of background risk. They first present a stochastic factor model with an additive background risk. Thereafter, they present a dynamic model of simultaneous (correlated) multiplicative background risk and additive background risk.

\subsection{Regret-Aversion}

Egozcue, Guo, and Wong (2015) study the optimal output of a competitive firm under price uncertainty. Instead of assuming a risk-averse firm, they assume that the firm is regret-averse. The authors find that optimal output under uncertainty would be lower than under certainty, and prove that the optimal output could increase or decrease as the regret factor varies.

Guo, Wong, $\mathrm{Xu}$, and Zhu (2015) investigate regret-averse firms' production and hedging behavior. The authors first show that the separation theorem is still alive under regret aversion by proving that regret aversion is independent of the level of optimal production. On the other hand, they find that the full-hedging theorem does not always hold under regret aversion as the regretaverse firms take hedged positions that can be different from those of risk-averse firms under certain situations. With more regret aversion, regret-averse firms will hold smaller optimal hedging positions in an unbiased futures market. Furthermore, contrary to conventional expectations, the authors show that banning firms from forward trading affects their production level in both directions.

\subsection{Contagion}

Wan and Wong (2001) provide a simple example of a refinancing game with incomplete information, where the lack of transparency is both necessary and sufficient for the propagation of local financial distress across disjoint financial networks. The authors emphasize that contagion is a very important topic in economics and finance. There are some tests for contagion, for example, the test developed by Fry, Martin and Tang (2010) and Fry-McKibbin and Hsiao (2015). The tests can be used for big data, medium data, and small data. 


\subsection{Covariance and Copulas}

Chebyshev's integral inequality, also known as the covariance inequality, is an important problem in economics, finance, marketing, management, and business decision making. Egozcue, Fuentes García, and Wong (2009) derive some covariance inequalities for monotonic and non-monotonic functions. The results can be useful in many applications in economics, finance, marketing, management, and decision making. Egozcue, Fuentes García, Wong, and Zitikis (2010) sharpen the upper bound of a Grüss-type covariance inequality by incorporating a notion of quadrant dependence between random variables and also using the idea of constraining the means of the random variables.

Egozcue, Fuentes García, Wong, and Zitikis (2011a) show that Grüss-type probabilistic inequalities for covariances can be considerably sharpened when the underlying random variables are quadrant dependent in expectation (QDE). The established covariance bounds not only sharpen the classical Grüss inequality, but also improve upon the recently derived Grüsstype bounds under the assumption of quadrant dependency (QD), which is stronger than QDE. The authors illustrate the general results with examples based on specially devised bivariate distributions that are QDE but not QD. Such results play important roles in decision making under uncertainty, and particularly in areas such as economics, finance, marketing, management, and insurance.

A number of problems in economics, finance and insurance rely on determining the signs of the covariances of two transformations of a random variable. The classical Chebyshev's inequality offers a powerful tool for solving the problem, but it assumes that the transformations are monotonic, which is not always the case in applications. For this reason, Egozcue, Fuentes García, Wong, and Zitikis (2011b) establish new results for determining the covariance signs and provide further insights into the problem. Unlike many previous research efforts, their method of analysis, which is probabilistic in nature, does not rely on the classical Hoffding's representation of the covariance, or on any of its numerous extensions and generalizations. 
Egozcue, Fuentes García, Wong, and Zitikis (2012) establish the smallest upper bound for the $p$ th absolute central moment over the class of all random variables with values in a compact interval. Numerical values of the bound are calculated for the first ten integer values of $p$, and its asymptotic behaviour derived as $p$ tends to infinity. In addition, they establish an analogous bound in the case of all symmetric random variables with values in a compact interval. Such results play an important role in a number of disciplines, including actuarial science, economics, finance, marketing, management, operations research, and reliability.

It is well known that quadrant dependent (QD) random variables are also quadrant dependent in expectation (QDE). The recent literature has offered examples that have rigorously established the fact that there are QDE random variables that are not QD. The examples are based on convex combinations of specially chosen QD copulas, namely one negatively QD and another positively QD. Egozcue, Fuentes García, Wong, and Zitikis (2013) establish general results that determine when convex combinations of arbitrary QD copulas lead to negatively or positively QD/QDE copulas. In addition to being an interesting mathematical exercise, the established results are helpful when modeling insurance and financial portfolios in practice.

\subsection{Stock Networks}

Xu, Wong, Chen, and Huang (2017) analyse the relationship among stock networks by focusing on the statistically reliable connectivity between financial time series, which accurately reflects the underlying pure stock structure. In order to do so, they first filter out the effect of the market index on the correlations between paired stocks, and then take a t-test based on the P-threshold approach to lessen the complexity of the stock network based on the P values. The authors

demonstrate the superiority of the performance in understanding network complexity by examining the Hong Kong stock market.

In comparison with other filtering methods, the authors find that the P-threshold approach extracts purely and significantly correlated stock pairs, which reflect the well-defined hierarchical structure of the market. In analyzing the dynamic stock networks with fixed-size moving windows, they show that three global financial crises, covered by the long-range time 
series, can be distinguishing as indicated from the network topological and evolutionary perspectives. In addition, they find that the so-called assortativity coefficient can manifest the financial crises, and hence can serve as a useful indicator of financial market developments.

\section{Statistical and Econometric Models}

Another useful and practical suggestion is to develop statistical and econometric models in the areas related to management information, decision sciences, economics, and finance, as well as cognate disciplines. After developing mathematical models, one might consider developing some related statistical and econometric models. Researchers have developed several econometrics papers related to management information, decision sciences, economics, and finance, and these will be discussed in the following sub-sections.

\subsection{Stochastic Dominance}

Ng, Wong, and Xiao (2017) develop tests for stochastic dominance by translating the inference problem of stochastic dominance into a proposal for testing parametric restrictions in quantile regressions. Specifically, the tests are variants of the one-sided Kolmogorov-Smirnoff statistic with a limiting distribution of the standard Brownian bridge. The procedures for obtaining the critical values of the proposed test statistics are provided. The simulation results show their superior small size and good power properties. The authors apply estimation to the NASDAQ 100 and S\&P 500 indices to investigate dominance relationship before and after major turning points. The empirical results show no evidence of arbitrage opportunities between the bear and bull markets.

Bai, Li, McAleer, and Wong (2015) derive the limiting process of stochastic dominance statistics for both risk averters and risk seekers where the underlying processes can be either dependent or independent. The authors take account of the dependency of the partitions and propose a bootstrap method to decide the critical points. In addition, they illustrate the applicability of the stochastic dominance statistics for both risk averters and risk seekers to analyse the dominance relationship between the Chinese and US stock markets for the entire sample period, as well as 
the sub-periods before and after the various financial crises, including the internet bubble and the recent sub-prime crisis.

The empirical findings could be used to draw inferences on the preferences of risk averters and risk seekers in investing in the Chinese and US stock markets. The results also enables an examination as to whether there are arbitrage opportunities in these markets, whether these markets are efficient, and an analysis of whether investors are rational.

Bai, Li, Liu and Wong (2011) develop new statistics for both PSD and MSD of the first three orders. These statistics provide tools to examine the preferences of investors with S-shaped utility functions in prospect theory, as well as for investors with RS-shaped investors. The authors also derive the limiting distributions of the test statistics to be stochastic processes, and propose a bootstrap method for determining the critical points of the tests, as well as proving the consistency of the bootstrap tests. They illustrate the practical applicability of the proposed statistics by evaluating the preferences of investors with corresponding S-shaped and RS-shaped utility functions vis-a-vis returns on iShares and returns on traditional stocks and Internet stocks before and after the Internet bubble.

Academics and practitioners can apply stochastic dominance tests in many different areas for big data, medium data, and small data. The extant literature for applying stochastic dominance tests includes Fong, Wong, and Lean (2005), Fong, Lean, and Wong (2008), Gasbarro, Wong, and Zumwalt (2007), Lean, Smyth, and Wong (2007), Qiao, Qiao, and Wong (2010), Lean, McAleer, and Wong (2010, 2015), Lean, Phoon, and Wong (2012), Chan, de Peretti, Qiao, and Wong (2012), Qiao, Clark, and Wong (2012), Qiao, Wong, and Fung (2013), Qiao and Wong (2015), Hoang, Lean, and Wong (2015), Hoang, Wong, and Zhu (2015), among many others.

\subsection{Portfolio Optimization}

The literature has seen the development of several new and interesting theoretical results on portfolio optimization. When the dimension of the data is large, the theoretical model of the classical MV portfolio optimization developed by Markowitz (1952) has been found to have 
serious problems in estimation. Substituting the sample mean and covariance matrix into the MV optimization procedure will result in a serious departure of the optimal return estimate, and the corresponding portfolio allocation estimate from their theoretic counterparts when the number of the assets can be large. We refer to the return estimate in this case as the "plug-in" return, and the corresponding estimate for the asset allocation as the "plug-in allocation."

Bai, Liu, and Wong (2009a) prove that this phenomenon is normal, and refer to the development as "over-prediction." In order to circumvent this over-prediction problem, the authors use a new method by incorporating the idea of the bootstrap into the theory of a large dimensional random matrix. They develop new bootstrap-corrected estimates for the optimal return and its asset allocation, and prove that these bootstrap-corrected estimates can correct analytically the overprediction and also drastically reduce the error. Moreover, they show that the bootstrap-corrected estimate of return and its corresponding allocation estimate are proportionally consistent with their counterpart parameters.

Bai, Liu, and Wong (2009a) propose a bootstrap-corrected estimator to correct the overestimation, but there is no closed-form solution for the estimator, so it has to be obtained by using a bootstrap approach. As a result, it seems to have been difficult for practitioners to adopt the estimate in reality. In order to circumvent this limitation, Leung, Ng, and Wong (2012) develop a new and convenient estimator for the optimal portfolio return based on an unbiased estimator of the inverse of the covariance matrix and its related terms, and derive explicit formulae for the estimator of the optimal portfolio return.

Bai, Li, McAleer, and Wong (2016) improve the estimation issue further by using the spectral distribution of the sample covariance. They develop the limiting behavior of the quadratic form with the sample spectrally corrected covariance matrix, and explain the superior performance to the sample covariance as the dimension increases to infinity with the sample size proportionally. Moreover, the authors deduce the limiting behavior of the expected return and risk on the spectrally corrected MV portfolio, and illustrate the superior properties of the spectral corrected MV portfolio with a practical example. 
In simulations, the authors compare the spectrally corrected estimates with the traditional and bootstrap-corrected estimates, and show the performance of the spectrally corrected estimates are the best in terms of portfolio returns, as well as for portfolio risk. They also compare the performance of the proposed estimation method with different optimal portfolio estimates for real S\&P 500 data.

Mroua, Abid, and Wong (2017) investigate the impact of the sampling errors on the optimal portfolio weights and on financial investment decisions. They propose a new methodology that combines the re-sampling method, stochastic optimization algorithm, and nonparametric stochastic dominance (SD) approach to analyze a stochastic optimal portfolio choice for risk-averse American investors who care about the benefits of domestic diversification relative to that of international diversification. Thereafter, the authors conduct a comparative analysis of various domestic and international diversification strategies to define a stochastic optimal choice.

The authors find that reducing the sampling error increases the dominance relationships among different portfolios which, in turn, can alter portfolio investment decisions. Although international diversification is preferred in some cases, the empirical results show that, for risk-averse US investors, in general, there is no difference between the alternative diversification strategies. They also find that stochastic diversification in domestic, global, European, Australasian, and Far Eastern markets delivers superior risk returns for US risk averters during the various crisis periods.

It is worth noting that portfolio optimization can be used for big data as well as medium and small data. In the theory developed by Bai, Liu, and Wong (2009a, b), Leung, Ng, and Wong (2012), and Li, Bai, McAleer, and Wong (2017) explain that their theory holds when the observations tend to infinity. Academics and practitioners can use portfolio optimization in their analysis for big data, medium data, and small data. The extant literature of using portfolio optimization in their interesting and practical empirical analyses includes Abid, Mroua, and Wong (2009, 1013), Abid, Leung, Mroua, and Wong (2014), Hoang, Lean, and Wong (2015), Hoang, Wong, and Zhu (2015), among many others. 


\subsection{Risk Measures}

Leung and Wong (2008) apply the technique of repeated measures design to develop the Multiple Sharpe ratio test statistic to test the hypothesis of the equality of the multiple Sharpe ratios. The authors also establish the asymptotic distribution of the statistic and its properties. In order to demonstrate the superiority of their proposed statistic over the traditional pairwise Sharpe ratio test, they illustrate their approach by testing the equality of Sharpe ratios for 18 iShares. Whereas the pairwise Sharpe ratio test show that the performance of all 18 iShares are indistinguishable, they reject the equality of the Sharpe ratios in each year, as well as for the entire sample. These empirical results suggest that the 18 iShares perform differently in each year, as well as for the entire sample period, with some outperforming other shares in the market.

Recent results in optimal stopping theory have shown that a 'bang-bang' (buy or sell immediately) style of trading strategy is, in some sense, optimal provided that the asset's price dynamics follow certain familiar stochastic processes. Wong, Wright, Yam, and Yung (2012) construct a reward-to-variability ratio (that is, the mixed Sharpe ratio) that is sufficient for implementation of this strategy. The use of this ratio for optimal portfolio selection is discussed, and empirical evidence for its variations over time is found. The performances of the 'bangbang' and 'buy-and-hold' trading strategies are compared, and the former is found to be significantly more profitable than the latter.

Bai, Wang, and Wong (2011) develop the mean-variance ratio statistic to test the equality of the mean-variance ratios, and prove that the proposed statistic is uniformly most powerful unbiased. In addition, the authors illustrate the applicability of the proposed test to compare the performances of stock indices. Thereafter, Bai, Hui, Wong, and Zitikis (2012) propose and develop mean-variance ratio (MVR) statistics for comparing the performance of prospects after the effect of the background risk has been mitigated. They investigate the performance of the statistics in large and small samples and show that, in the non-asymptotic framework, the MVR statistic produces a uniformly most powerful unbiased (UMPU) test. 
The authors discuss the applicability of the MVR test in the case of large samples, and illustrate its superiority in the case of small samples by analyzing stock returns in Korea and Singapore after the impact of the American stock returns (which are viewed as background risk) has been deducted. They find, in particular, that when samples are small, the MVR statistic can detect differences in asset performances while the Sharpe ratio test, which is the mean-standarddeviation-ratio statistic, may not be able to do so.

Academics and practitioners can apply different risk measures, estimators and test statistics in many different areas for big data, medium data, and small data. The extant literature in applying different risk measures, estimators and test statistics includes Gasbarro, Wong, and Zumwalt (2007), Lean, Smyth, and Wong (2007), Lean, McAleer, and Wong (2010, 2015), Lean, Phoon, and Wong (2012), Chan, de Peretti, Qiao, and Wong (2012), Qiao, Clark, and Wong (2012), Bai, Phoon, Wang, and Wong (2013), Qiao, Wong, and Fung (2013), Qiao and Wong (2015), Hoang, Lean, and Wong (2015), Hoang, Wong, and Zhu (2015), among many others.

\subsection{Testing Investors’ Behavorial Models}

Lam, Liu, and Wong $(2008,2010)$ develop a Bayesian model for analysing excess volatility, short-term underreaction and long-term overreaction, while Guo, McAleer, Wong, and Zhu (2017) extend their model to excess volatility, short-term underreaction and long-term overreaction during financial crises. Fabozzi, Fung, Lam, and Wong (2013) develop three tests of the magnitude effect of short-term underreaction and long-term overreaction.

It is worth noting that testing investors' behavorial models developed by Lam, Liu, and Wong (2008, 2010), and Guo, McAleer, Wong, and Zhu (2017), can be used for big data, medium data and small data. Fabozzi, Fung, Lam, and Wong (2013) developed three tests, and use S\&P data to test for the magnitude effect of short-term underreaction and long-term overreaction. Academics and practitioners can apply the convenient tests developed in Fabozzi, Fung, Lam, and Wong (2013) for a wide range of practical situations, such as data from international markets. The observations could be taken over a large number of years, so that the tests can be used especially for big data, as well as medium and small data. 
Wong, Chow, Hon, and Woo (2017) conduct a questionnaire survey to examine whether the theory developed by Lam, Liu, and Wong (2008, 2010), and Guo, McAleer, Wong, and Zhu (2017), holds empirically by examining the conservative and representative heuristics by Hong Kong small investors who adopt momentum and/or contrarian trading strategies. It is worth noting that academics and practitioners could conduct a questionnaire survey on big data as well as medium and small data cases in this developing area.

\subsection{Economic and Financial Indicators}

The literature has developed some financial indicators and applied some economic indicators to examine several important economic issues. For example, Wong, Chew, and Sikorski (2001) develop a new financial indicator to test the performance of stock market forecasts by using E/P ratios and bond yields. They also develop two test statistics to use the indicator, and illustrate the practicality of the tests in several stock markets. The empirical results show that the forecasts generated from the indicator would enable investors to escape most of the crashes, and catch most of the bull runs.

The trading signals provided by the indicator can generate profits that are significantly better than the buy-and-hold strategy. Exploring the characteristics associated with the formation of bubbles that occurred in the Hong Kong stock market in 1997 and 2007, and the 2000 dot-com Nasdaq bubble, McAleer, Suen, and Wong (2016) establish trading rules that not only produce returns that are significantly greater than buy-and-hold strategies, but also produce greater wealth as compared with TA strategies without trading rules.

Chong, Cao, and Wong (2017) develop a new market sentiment index for the Hong Kong stock market, one of the largest stock markets in the world, by using the turnover ratio, short-selling volume, money flow, HIBOR, and returns of the US and Japanese markets, the Shanghai and Shenzhen Composite indices. Thereafter, the authors incorporate the threshold regression model with the sentiment index as a threshold variable to capture the state of the Hong Kong stock market. It can be established empirically that the trading rule which sells (buys) the HSI or 
S\&P/HKEx LargeCapIndex when the sentiment index is above (below) the upper threshold value can beat the buy-and-hold strategy.

Sethi, Wong, and Acharya (2017) examine the sectoral impact of disinflationary monetary policy by calculating the sacrifice ratios for several OECD and non-OECD countries. Sacrifice ratios calculated through the episode method reveal that disinflationary monetary policy has a differential impact across three sectors in both OECD and non-OECD countries. Of the three sectors, the industry and service sectors show significant output loss due to a tight monetary policy in OECD and non-OECD countries.

The agricultural sector shows a differential impact of disinflation policy, In particular, the empirical analysis shows a negative sacrifice ratio in OECD countries, indicating that output growth is not significantly affected by a tight monetary policy, while non-OECD countries yield positive sacrifice ratios, suggesting that the output loss is significant. Furthermore, it is observed that sacrifice ratios calculated from aggregate data are different from ratios that are calculated from sectoral data.

Financial indicators and economic indicators can be used for big data, medium data, and small data. For example, Wong, Chew, and Sikorski (2001) use their indicator to test for markets from the USA, UK, Japan, Germany, and Singapore. This is not especially big data. Academics and practitioners could use the indicators to test for international stock markets. They can use it to test not only for stock markets, but also for any financial products, and so it can be used to test in cases of big data.

Similarly, Sethi, Wong, and Acharya (2017) apply the sacrifice ratios to examine the sectoral impact of disinflationary monetary policy for several OECD and non-OECD countries, which would not necessarily be regarded as an application to big data. However, academics and practitioners can apply the sacrifice ratios to examine the sectoral impact of disinflationary monetary policy for all the countries in the world, this would lead to a much bigger data set that could encompass big data. 


\subsection{Technical Analysis}

The new financial indicator introduced by Wong, Chew, and Sikorski (2001) to test the performance of stock market forecasts can be classified as technical analysis. Substantial research has been undertaken in technical analysis. For example, Wong, Manzur, and Chew (2003) use technical analysis to signal the timing of stock market entry and exit. The authors develop test statistics to test the performance of the most established of the trend followers, namely the Moving Average (MA), and the most frequently used counter-trend indicator, namely the Relative Strength Index. Using data for Singapore, the empirical results indicate that the indicators can be used to generate significantly positive returns. It is also found that member firms of the Singapore Stock Exchange tend to enjoy substantial profits by applying technical indicators.

Wong, Du, and Chong (2005) evaluate the profitability of applying technical analysis that signals the entry and exit from the stock market in three Greater Chinese stock markets, namely the Shanghai, Hong Kong and Taiwan Stock Exchanges. Applying the trading signals generated by the MA family to these inter-connected markets shows that significantly positive returns can be generated, which outperform the buy-and-hold strategy. The cumulative wealth obtained also surpasses that of the buy-and-hold strategy, regardless of transaction costs. In addition, the authors examine the performance of the MA family before and after the 1997 Asian Financial Crisis, and find that the MA family works well in both sub-periods, as well as in different market conditions of bull runs, bear markets, and mixed markets.

The finding that technical analysis can forecast the directions of these markets implies that the three Greater China stock markets are not efficient. Lam, Chong, and Wong (2007) examine whether a day's surge or plummet in stock prices can serve as a market entry or exit signal. Returns of five trading rules based on 1-day and intraday momentum are estimated for major world stock indices, and it is found that the trading rules perform well for the Asian indices, but not for stock indices in Europe and USA. 
Kung and Wong (2009a) investigate whether these measures have led to lower profitability for those investors who employ technical rules for trading stocks. Their results show that the three trading rules consistently generate higher annual returns for 1988-1996 than those for 19992007. Furthermore, these measures generally perform better than the buy-and-hold (BH) strategy for 1988-1996, but perform no better than the BH strategy for 1999-2007.

These empirical findings suggest that the efficiency of the Singapore stock market has been considerably enhanced by the measures implemented after the various financial crises. Kung and Wong (2009b) use two popular technical trading rules to assess whether the gradual liberalization of Taiwan's securities markets has improved the efficiency of its stock market. The empirical results show that the two rules have considerable predictive power for 1983-1990, become less predictive for 1991-1997, and cannot predict the market usefully for 1998-2005. These results indicate that the efficiency of the Taiwan stock market has been greatly enhanced by the liberalization measures that have been implemented over the past 20 years.

The above studies examine technical analysis for reasonably big data. In addition, academics and practitioners can apply technical analysis to examine the performance of a larger number of stock markets, as well as other financial markets with bigger data sets.

\subsection{Cost of Capital}

Gordon and Shapiro (1956) develop the dividend yield plus growth model for individual firms, while Thompson (1985) improves the theory by combining the model with an analysis of previous dividends to estimate the cost of capital and its 'reliability'. Thompson and Wong (1996) extend the theory by obtaining estimates for the cost of equity capital and its reliability. Wong and Chan (2004) extend the theory further by developing estimators for the reliability, and prove that the estimators are consistent. It is worth noting that estimation for the cost of equity capital and its reliability can be used for big data, medium data, and small data, making it a worthwhile method in practice.

\subsection{Robust Estimation}


Bian and Dickey (1996) develop a robust Bayesian estimator for the vector of regression coefficients using a Cauchy-type g-prior. This estimator is an adaptive weighted average of the least squares estimator and the prior location, and has substantial robustness properties with respect to flat-tailed sampling distribution. Wong and Bian (1997) develop an alternative approach to estimate regression coefficients, while Wong and Bian (2000) introduce the robust Bayesian estimator developed by Bian and Dickey (1996) to the estimation of the Capital Asset Pricing Model (CAPM), in which the distribution of the error component is well known to be flat-tailed. In order to support their proposal, the authors apply both the robust Bayesian estimator and the least squares estimator in the simulation of the CAPM, and in the analysis of the CAPM for US annual and monthly stock returns.

Their simulation results show that the Bayesian estimator is robust and superior to the least squares estimator when CAPM is contaminated by large normal and/or non-normal disturbances, especially by Cauchy disturbances. In their empirical study, the authors find that the robust Bayesian estimator is uniformly more efficient than its least squares counterpart in terms of the relative efficiency of one-step ahead forecast mean square error, especially in small samples. They introduce the robust Bayesian estimator developed by Bian and Dickey (1996) as this robust Bayesian estimator is adaptive, and markedly robust with respect to a flat-tailed sample distribution. However, to date few papers seem to have used this estimator in practice.

The estimator is adaptive and robust in the sense that, if the sample is adequate, that is, without extreme observations and outliers, the estimator will concentrate on using the sample information. On the other hand, if there are many outliers in the sample, the robust Bayesian estimator will rely on using information from the prior. To date, the estimator developed in Bian and Dickey (1996) seems to be the only approach that has this feature, and so its use is recommended. It is worth noting that the robust Bayesian estimator can be used for the trilogy of big data, medium data, and small data.

\subsection{Unit Roots, Cointegration, Causality Tests, and Nonlinearity}


The literature has published a number of tests that are related to unit roots, cointegration, causality, and nonlinearity, which will be discussed in the following sections.

Tiku and Wong (1998) develop a unit root test to analyse if data follow an AR(1) model. The authors suggest use of the three moment chi-square and four moment $\mathrm{F}$ approximations to test for a unit root in an AR(1) model when the innovations arise from one of a wide family of symmetric Student's t distributions.

In cointegration theory, vector error-correction models (VECMs) have become an important means of analysing cointegrating relations. Usual full-order VECMs assume all non-zero entries in the coefficient matrices. However applications of VECMs to economic and financial time series data have revealed that zero entries are indeed possible. If indirect causality or Granger non-causality exists among the variables, the use of a full-order VECM may conclude incorrectly that there is Granger causality among the variables.

In addition, the statistical and numerical accuracy of the cointegrating vectors estimated in a misspecified full-order VECM will be doubtful. It is argued that the zero-non-zero (ZNZ) patterned VECM is a more straightforward and effective means of testing for both indirect causality and Granger non-causality. Penm, Terrell and Wong (2003) present simulations and an empirical application that demonstrate the usefulness of the ZNZ-patterned VECM.

Lam, Wong, and Wong (2006) develop some properties for the autocorrelation of the $k$-period returns for the general mean reversion (GMR) process, in which the stationary component is not restricted to the AR(1) process but takes the form of a general ARMA process. The authors derive several properties of the GMR process and three new nonparametric tests that compare the relative variability of returns over different horizons to validate the GMR process as an alternative to a random walk process. They also examine the asymptotic properties of the tests, which can be applied to identify random walk models from the GMR processes.

The traditional linear Granger causality test has been used widely to examine linear causality among several time series processes in both bivariate and multivariate settings. Hiemstra and 
Jones (1994) develop a nonlinear Granger causality test in a bivariate setting to investigate the nonlinear causality between stock prices and trading volume. Bai, Wong, and Zhang (2010) extend their work by developing a nonlinear causality test in multivariate settings. Bai, Li, Wong, and Zhang (2011) discuss linear causality tests in multivariate settings, and thereafter develop a nonlinear causality test in multivariate settings.

A Monte Carlo simulation is conducted to demonstrate the superiority of the newly proposed multivariate test over its bivariate counterpart. In addition, the authors illustrate the applicability of the proposed test to analyse the relationships among different Chinese stock market indices. Bai, et al. (2017) improve the properties of the statistics by re-estimating the probabilities and reestablishing the central limit theorem of the new test statistic. They also conduct simulations to show that the new estimates are consistent, and that the new test has low size and high power. Chow, Cunado, Gupta, and Wong (2017) modify the multivariate nonlinear causality test so that it can also be used for panel data models.

Hui, Wong, Bai, and Zhu (2017) propose a simple and efficient method to examine whether a time series Yt possesses any nonlinear features by testing dependence that might remain in the residuals after fitting Yt using a linear model. The advantage of the proposed nonlinearity test is that it is not necessary to know the exact nonlinear features and the detailed nonlinear forms of the stochastic process underlying Yt. The procedure can also be used to test whether the hypothesized model, including linear and nonlinear components, is appropriate if the residuals of the model can be obtained from the estimated model. Simulation experiments show that the new test is stable and powerful. The authors apply the new statistic to test whether there are any nonlinear features in sunspot data, and whether the S\&P 500 index follows a random walk. The conclusions drawn regarding the new test are consistent with the outcomes from other tests.

Academics and practitioners can apply unit root, cointegration, causality, and nonlinearity tests in many different areas for big data, medium data, and small data. The extant literature for applying unit root, cointegration, causality, and nonlinearity tests includes Wong, Penm, Terrell, and Lim (2004), Wong, Khan, and Du (2006), Qiao, Liew, and Wong (2007), Foo, Wong, and Chong (2008), Qiao, Smyth, and Wong (2008), Qiao, Chiang, and Wong (2008), Chiang, Qiao, 
and Wong (2009), Qiao, McAleer, and Wong (2009), Qiao, Li, and Wong (2011), Vieito, Wong, and Zhu (2015), among many others.

\subsection{Confidence Intervals}

Homm and Pigorsch (2012) use the Aumann and Serrano index to develop a new economic performance measure (EPM), which is well known to have advantages over other measures. Niu, Guo, McAleer, and Wong (2017) extend the theory by constructing a one-sample confidence interval of EPM, and construct confidence intervals for the differences in EPMs for two independent samples. The authors also derive the asymptotic distribution for EPM and the difference in two EPMs when the samples are independent. They conduct simulations to show that the proposed theory performs well for one and two independent samples.

Simulations show that the proposed approach is robust in the dependent case. The theory developed is used to construct both one-sample and two-sample confidence intervals of EPMs for Singapore and US stock indices. It is noted that confidence interval estimation could be used for big data, medium data, and small data. It is also worth noting that the theory of confidence intervals for EPM developed by Niu, Guo, McAleer, and Wong (2017) can be used to develop the theory of confidence intervals for any risk measure or economic indicator which, in turn, could be used to construct confidence intervals for big data, medium data, and small data.

\subsection{Mixture of Distributions Model}

Andersen (1996) introduces a modification of the mixture of distributions model based on microstructure arguments. Based on a small sample of five stocks, he infers that a modified mixture of distributions (MMD) model adequately captures the joint behavior of trading volume and volatility. Fong and Wong (2006) re-examine this claim using a larger sample of twenty-two stocks and two sample periods. Their tests show that 59\% of the sample rejects the MMD model in the period 1973-1991, which is the same period as examined in Andersen (1996). The empirical results for the second period (1993-1999) are more supportive of MMD, especially for the number of trades, although nearly one-third of the sample still rejects the MMD. The authors 
conclude that further investigation is needed before the general validity of the MMD can be established.

\subsection{Repeated Time Series}

Wong and Miller (1990) develop a theory and methodology for repeated time series (RTS) measurements for an autoregressive integrated moving average-noise (ARIMAN) process. The theory enables academics and practitioners to relax the normality assumption in the ARIMAN model and to identify models for each component process of the time series. The authors also discuss the properties, estimation, and forecasting of RTS ARIMAN models, and provide illustrative examples. Wong, Miller, and Shrestha (2001) extend the RTS model where the fundamental component follows an ARMA process. In the model, the error variance and the number of repetitions are allowed to change over time. It is shown that the model is identified, and the maximum likelihood estimator is derived using the Kalman filter technique.

\subsection{Multinomial Distribution Model}

Matsumura, Tsui, and Wong (1990) extend the procedure developed in Tsui, Matsumura, and Tsui (1985), which is based on a multinomial distribution model within the dollar-unit sampling

framework, with a Dirichlet prior distribution. The extended model and a different Dirichlet prior are used in the analysis to generate upper and lower bounds, as well as two-sided confidence intervals, for situations in which both understatement and overstatement errors are possible. The simulation results indicate that the calculated confidence levels of the proposed estimates are usually close to or greater than the nominal levels in repeated sampling for the populations that are investigated.

\subsection{Other Econometric Models/Tests}

Researchers have been developing a wide range of alternative econometric models and statistical tests in the literature, some of which will be presented and discussed in the following section. 
Tiku, Wong, Vaughan, and Bian (2000) consider AR(q) models for time series data with nonnormal innovations that are represented by a member of a wide family of symmetric Student's $t$ distributions. As the ML (maximum likelihood) estimators are intractable, the authors derive the MML (modified maximum likelihood) estimators of the parameters, and show that they are efficient. They use the estimators for hypothesis testing, and show that the resulting tests are robust and powerful.

Tiku, Wong, and Bian (1999a) extend the previous work by considering $\operatorname{AR}(q)$ models in time series with asymmetric innovations that are represented by two families of distributions, namely

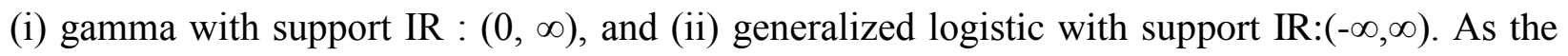
ML (maximum likelihood) estimators are intractable, the authors derive the MML (modified maximum likelihood) estimators of the parameters and show that they are efficient and computationally straightforward. The authors investigate the efficiency properties of the classical LS (least squares) estimators, and find that the efficiency relative to the proposed MML estimators is low.

Tiku, Wong, and Bian (1999b) estimate the coefficients in a simple regression model with autocorrelated errors, where the underlying distribution is assumed to be symmetric, namely one of the Student's t family for illustrative purposes. Closed-form estimators are obtained, and are shown to be efficient and robust. Wong and Bian (2005) extend the results to the case where the underlying distribution is a generalized logistic distribution. The generalized logistic distribution family represents very wide range of skewed distributions, from highly right skewed to highly left skewed. Analogously, the authors develop MML estimators as the ML (maximum likelihood) estimators are intractable for generalized logistic data. The authors analyse the asymptotic properties of the proposed estimators and conduct simulation experiments to examine the finite sample properties of the estimators

Bian and Dickey (1996) develop a robust Bayesian estimator for the vector of regression coefficients using a Cauchy-type g-prior. This estimator is an adaptive weighted average of the least squares estimator and the prior location, and is robust with respect to a fat-tailed sample distribution. Wong and Bian (2000) introduce the robust Bayesian estimator to the estimation of 
the Capital Asset Pricing Model (CAPM), in which the distribution of the error component is well known to be fat-tailed.

In order to support their proposal, the authors apply both the robust Bayesian estimator and the least squares estimator in the simulation of the CAPM and in the analysis of the CAPM for US annual and monthly stock returns. The simulation results show that the Bayesian estimator is robust and superior to the least squares estimator when CAPM is contaminated by large normal and/or non-normal disturbances, especially by Cauchy disturbances. In the empirical analysis, the authors find that the robust Bayesian estimator is uniformly more efficient than the least squares estimator in terms of the relative efficiency of one-step ahead forecast mean square errors, especially in small samples.

Bian, McAleer, and Wong (2013) develop a modified maximum likelihood (MML) estimator for the multiple linear regression model with underlying Student's t distribution. The authors obtain the closed-form solution of the estimators, derive the asymptotic properties, and demonstrate that the MML estimator is more appropriate for estimating the parameters of CAPM by comparing its performance with least squares estimators (LSE) on monthly returns of US portfolios. The empirical results reveal that the MML estimators are more efficient than their least squares counterparts in terms of the relative efficiency of the one-step-ahead forecast mean square errors in small samples.

Bian, McAleer, and Wong (2011) develop a new test, the trinomial test, for pairwise ordinal data samples to improve the power of the sign test by modifying its treatment of zero differences between observations, thereby increasing the use of sample information. The simulations demonstrate the power superiority of the proposed trinomial test statistic over the sign test in small samples in the presence of tie observations. The authors show that the proposed trinomial test has substantially higher power than the sign test in large samples, and also in the presence of tie observations, as the sign test ignores information from observations that result in ties.

It is worth noting that all of the above estimation and testing methods could be used for big data, medium data, and small data. 


\section{Simulations}

After developing statistical theories for Big Data, Computational Science, Economics, Finance, Marketing, Management, and Psychology, academics and practitioners could consider conducting simulations to examine whether the estimators or statistics in their theories on estimation and hypothesis testing have small size and high power in finite samples. We have been conducting simulations to examine whether the new estimators are consistent, and if the statistics in the new theories on hypothesis testing have adequately small size and high power in finite samples. For example, Tiku and Wong (1998) conduct simulations to examine whether their unit root test has small size and high power.

Tiku, Wong, and Bian (1999) consider AR(q) models in time series with asymmetric innovations represented by the gamma and generalized logistic distributions. They derive the MML (modified maximum likelihood) estimators of the parameters and show that they are efficient. Thereafter, they conduct simulations to examine whether their estimators have small size and high power in finite samples. Tiku, Wong, and Bian (1999a) develop the theory to estimate the coefficients in a simple regression model with autocorrelated errors with the Student's $t$ distribution.

Thereafter, the authors conduct simulations to examine whether their estimators have small size and high power in finite samples. Tiku, Wong, Vaughan, and Bian (2000) consider AR(q) models for time series data with non-normal innovations that are represented by a member of a wide family of symmetric Student's t distributions. The authors derive the MML estimators of the parameters and show that they are efficient, use the estimators for hypothesis testing, and conduct simulation experiments to show that the resulting tests are robust and powerful.

After checking that the new theories on estimation and hypothesis lead to consistent estimators,

and small size and high power in finite samples, respectively, academics and practitioners could then apply the theories to analyse some interesting and practical issues arising in the presence of big data, medium data, and small data. 


\section{Empirical Studies}

After developing theoretical models and econometric and statistical models to estimate the parameters in the associated models, academics and practitioners could then apply their theories to analyse some interesting and practical issues in the six disciplines and associated cognate disciplines.

\subsection{Applications in Economics and Finance}

Readers may refer to Chang, McAleer, and Wong (2016a) for applying the theoretical models and econometric and statistical models and tests to behavioral, financial, health, and medical economics; to Chang, McAleer, and Wong (2016b) for applying the theoretical models and econometric and statistical models and tests to informatics, data mining, econometrics and financial economics; to Chang, McAleer, and Wong (2016c) for applying the theoretical models and econometric and statistical models and tests to management science, economics and finance; and to Chang, McAleer, and Wong (2017a) for applying the theoretical models and econometric and statistical models and tests to management information, decision sciences, and financial economics.

Chang, McAleer, and Wong (2017b) apply the theoretical models and econometric and statistical models to big data, computational science, economics, finance and management. Academics and practitioners could apply their theories to other financial economic problems. For example, Batai, Chu, Lv, and Wong (2017) examine the factors that maintain a long-run equilibrium, short-run impact, and causality with the exchange rate of Mongolia over China to shed light on exchange rate determination. Cointegration analysis as applied to the problem shows that, in the long run, the gross domestic product (GDP) of China and the index of world price have significantly positive effects, while Mongolia's GDP and the Shanghai stock index have significantly negative effects on the Mongolian exchange rate. 
The authors reveal the existence of short-run dynamic interactions and strongly significant multivariate linear and nonlinear causality from all the explanatory variables to the Mongolian exchange rate. In addition, they observe that there is strong linear causality from each of the respective GDP of Mongolia and China and the index of world price to the Mongolian exchange rate, but not from the index of world price. Moreover, there is strongly significant nonlinear causality from the Shanghai stock index to the Mongolian exchange rate, and weakly significant nonlinear causalities from both the GDP of China and the index of world price to the Mongolian exchange rate, but not from Mongolia's GDP. The empirical findings should be useful to investors, manufacturers, and international trading firms for investment decision making, and also to policy makers for their decisions regarding both monetary and fiscal policies that could affect the Mongolian exchange rate.

Bouri, Gupta, Wong, and Zhu (2017) examine the role of wine investment within a portfolio of different assets (US/UK equities, bonds, gold, and housing) by considering a rich methodology based, among others, on the mean-variance and stochastic dominance approaches. The authors discover that wine is the best investment among all individual assets under investigation. It seems that investors prefer to invest in with-wine portfolios than without-wine portfolios in order to gain higher expected utility when short sales are not allowed. However, investors are indifferent between portfolios with-wine and without-wine when short selling is allowed.

In addition, with-wine portfolios generally either dominate individual assets or are indifferent from individual assets. Interestingly, the with-wine portfolios first-order stochastically dominates housing in both long-only and short-allowed strategies, pointing towards market inefficiency and therefore the possibility of expected arbitrage opportunities. Finally, the authors reveal that investors prefer the low risk with-wine portfolios to the equal-weighted portfolio, but are indifferent between the high risk with-wine portfolios and the naïve portfolio for both long-only and short-allowed strategies. The empirical findings can be used by investors in their investment decision making processes, and reveal the possibility of earning abnormal returns when wine is included in an investment portfolio. 
Manzur, Wong, and Chau (1999) use a new measure of real exchange rates as an indicator of international competitiveness. This new measure involves defining all prices and exchange rates on an appropriately weighted basket of currencies rather than on a single currency. The new measure is applied to data for Japan, Korea, Thailand, Malaysia and Singapore. For comparative purposes, the authors calculate real exchange rates based on purchasing power parity (PPP) for these countries. In order to check for the relative performance of the two measures, cointegration tests are used.

The empirical results indicate that the new measure tends to be closely related with export growth for the sample countries, while the PPP-based measure is not. Moreover, the PPP-based real exchange rates tend to understate the measures of competitiveness for these countries. This empirical result has important implications in terms of the levels of the exchange rates for these countries, as well as for the well-known Balassa hypothesis.

Phang and Wong (1997) find that interest rates, income growth rates and the supply of housing have not played a statistically significant role in the determination of private housing prices in Singapore between 1975 and 1994. Instead, private housing prices in Singapore are found to be highly correlated with the prices for public sector-built housing. Moreover, the timing of government policies relating to the use of compulsory savings for private housing finance purposes, the liberalisation of rules on public housing ownership criterion and for housing finance had significant impacts on private housing prices.

Phang, Wong, and Chia (1996) examine the policy processes behind Singapore's car quotas. The policy, when filtered through the market mechanism, had a number of unintended consequences. The public's disatisfaction with certain features and 'loopholes' of the system resulted in many changes to the rules. The effects of recent measures to curb speculation on quota premiums are also evaluated, and problems with Singapore's Weekend Car Scheme are also discussed. The lesson for transport policy makers elsewhere is that, in attempting to deal with the road congestion problem through car ownership policies, an asset market for vehicles should be taken into account to enable a more comprehensive analysis. 
The Indian stock market is one of the earliest in Asia, having been in operation since 1875, but it remained largely outside the global integration process until the late 1980s. A number of developing countries, together with the International Finance Corporation and the World Bank, took steps in the 1980s to establish and revitalize their stock markets as an effective way of mobilizing and allocating finance. In line with global trends, the reform of the Indian stock market began with the establishment of the Securities and Exchange Board of India in 1988.

Wong, Agarwal, and Du (2004) empirically investigate the long-run equilibrium relationship and short-run dynamic linkage between the Indian stock market and the stock markets in major developed countries, such as USA, UK and Japan, after 1990 by examining the Granger causality relationship and the pairwise, multiple and fractional cointegration between the Indian stock market and the stock markets from the three developed markets.

The authors conclude that the Indian stock market is integrated with mature markets and is sensitive to the dynamics in these markets in the long run. In the short run, both US and Japan Granger cause the Indian stock market, but not vice-versa. In addition, the authors find that the Indian stock index and the mature stock indices form a fractionally cointegrated relationship in the long run with a common fractional, nonstationary component, and find that the Johansen estimation and testing method is the most powerful in revealing the underlying cointegration relationship.

Xiao, Brooks, and Wong (2009) explore the relationship between volume and volatility in the Australian Stock Market in the context of a generalized autoregressive conditional heteroskedasticity (GARCH) model. In contrast to other studies that examine only the interaction of GARCH and volume effects on a small number of stocks, the authors examine the effects on the entire data set that is available for the Australian All Ordinaries Index. They also emphasize the impact of firm size and trading volume as important explanatory factors. It is found that GARCH model testing and estimation is impacted by firm size and trading volume.

Specifically, the empirical analysis produces the following major findings. First, daily trading volume, used as a proxy for information arrival time, is typically shown to have significant 
explanatory power regarding the variance of daily returns. Second, the actively traded stocks, which may have a larger number of information arrivals on a daily basis, have a larger impact of volume on the variance of the daily returns. Third, the authors find that low trading volume and small firms lead to a higher persistence of GARCH effects in the estimated models. Fourth, unlike the elimination effect for the top most active stocks, in general, the elimination of both autoregressive conditional heteroskedasticity (ARCH) and Generalised ARCH (GARCH) effects, by introducing the volume variable on all other stocks, on average, is not as much as that for the top most active stocks. Fifth, the elimination of both ARCH and GARCH effects by introducing the volume variable is higher for stocks in the largest volume and/or the largest market capitalization quartile group.

The empirical findings imply that the earlier findings in the literature were not a statistical mistake, and that, unlike most perceived anomalies, the volume effect on volatility is not likely to be eliminated after its discovery. In addition, the empirical findings reject the pure random walk hypothesis for stock returns.

Current integration and co-movements among international stock markets has been boosted by increased globalization of the world economy, and profit-chasing capital surfing across borders. With a reputation as the fastest growing economy in the world, China's stock market has continued gaining momentum in recent years, and incurred growing attention from academics, as well as practitioners. Taking into account economic and geographical considerations, the US and Hong Kong are considerably the most comparable stock markets to China. The usual vector error correction model (VECM) could overlook the long memory feature of the cointegration residual series which can, in turn, exert bias on the resulting estimators and statistical inference.

In order to overcome its limitations, Zheng, Chen, and Wong (2009) use a fractionally integrated VECM (FIVECM) to investigate the long-term cointegration relationships that bind China's stock market to the aforementioned stock markets. In addition, by augmenting the FIVECM with a multivariate GARCH model, the returns transmission and volatility spillovers between market return series were revealed simultaneously. The empirical results show that China's stock market is fractionally cointegrated with the two markets. Moreover, it also appears that China's stock 
market has stronger ties with the neighboring Hong Kong market than with the world superpower financial market, namely the USA.

\subsection{Applications in Psychology, Marketing, and Management}

In this section, we discuss in some detail several applications to marketing, management, and psychology. In the above section, we discussed Chang, Wong, Teo, and Fam (1997), Chang, Wong, and Teo (2000), Chang, Wong, and Koh (2003), Liao, Shi, and Wong (2012, 2014), Liao and Wong (2008), Moslehpour, Wong, Aulia, and Pham (2017), Moslehpour, Wong, Lin, and Huyen (2017). For further detailed analysis, the interested reader is referred to Chang, McAleer, and Wong (2016a, c, 2017a, b).

In what follows, we will discuss some other relevant papers that may make a significant contribution to understanding perceptions, personality and technology data in the future. For example, Moslehpour, Pham, Wong, and Bilgiçli (2017) propose a new model by partially combining personality traits (PT) and the Technology Acceptance Model (TAM) attributes to examine the influences of personality characteristics (such as conscientiousness and openness), and perception of technology (such as perceived usefulness and perceived ease of use) on epurchase intentions.

The authors use a truncated sampling technique and survey questionnaire to target the sample of Taiwanese online consumers and collect data. They find that consciousness (CON) (personality attribute) significantly influences perceived usefulness (PU) (technology perception attributes), perceived ease of use (PEOU) (technology perception attributes), and openness to experience (OPE) (personality attribute). PU, PEOU, and OPE have significant impacts on e-purchase intentions (INT). PEOU has the strongest positive impact on (INT). In addition, PU, PEOU, and OPE combined together mediate the relationship between CON and INT. Further post hoc analysis of mediation shows that both PU and PEOU are sustainable mediators. However, OPE is not a significant mediator.

\section{Concluding Remarks}


In this paper, we discussed a number of theoretical models in the areas of utility functions, stochastic dominance for risk averters and risk seekers, stochastic dominance for investors with S-shaped and reverse S-shaped utility functions, stochastic dominance theory for investors with both risk-averse and risk-seeking components, almost stochastic dominance, portfolio optimization, Sharpe ratio, Value-at-Risk (VaR), and Conditional VaR (CVaR), Omega ratio, first- and high-order risk measures, cost of capital, behavorial models, indifference curves, diversification, two-moment decision model, dynamic models with background risk, regretaversion, contagion, covariance and copulas, and stock network, among other topics.

We discussed several econometric and statistical models in the areas of stochastic dominance, portfolio optimization, risk measures, testing investors' behavorial models, analysed economic and financial indicators, technical analysis, cost of capital, unit roots, cointegration, causality tests, nonlinearity, confidence intervals, mixtures of distributions model, repeated time series, multinomial distribution model, and other econometric models and tests.

The paper also discussed research in simulation, and several applications in decision sciences, economics, finance, business, computing, and big data, as well as in cognate disciplines. Finally, we have discussed why analyzing big data is different from analyzing medium data or small data. Interested readers may wish to refer to Chang, McAleer, and Wong (2017b) for a discussion as to why analysing big data is different from analyzing medium data or small data. 


\section{References}

Abid, F., Leung, P.L., Mroua, M., Wong, W.K., 2014, International diversification versus domestic diversification: Mean-variance portfolio optimization and stochastic dominance approaches, Journal of Risk and Financial Management, 7(2), 45-66.

Abid, F., Mroua, M., Wong, W.K., 2013, Should Americans invest internationally? The meanvariance portfolios optimization and stochastic dominance approaches, Risk and Decision Analysis, 4(2), 89-102.

Aboudi, R., Thon, D., 1994, Efficient algorithms for stochastic dominance tests based on financial market data, Management Science, 40(4), 508-515.

Alghalith, M., Guo, X., Niu, C.Z., Wong, W.K., 2017, Input demand under joint energy and output prices uncertainties, Asia Pacific Journal of Operational Research, 34, 1750018.

Alghalith, M., Guo, X., Wong, W.K., Zhu, L.Z., 2016, A general optimal investment model in the presence of background risk, Annals of Financial Economics, 11(1), 1650001.

Alghalith, M., Niu, C.Z., Wong, W.K., 2017, The impacts of joint energy and output prices uncertainties in a mean-variance framework, Theoretical Economics Letters, 7, 1108-1120.

Andersen, T.G., 1996, Return volatility and trading volume: an information flow interpretation of stochastic volatility, Journal of Finance, 51, 169-204.

Bai, Z.D., Guo, X., Li, H., Wong, W.K., 2017, Stochastic dominance with applications in economics, finance, and income inequality, World Scientific Publishing (forthcoming).

Bai, Z.D., Hui, Y.C., Jiang, D.D., Lv, Z.H., Wong, W.K., Zheng, S.R. 2017, A new test of multivariate nonlinear causality, PLOS ONE, forthcoming.

Bai, Z.D., Hui, Y.C., Wong, W.K., Zitikis, R., 2012, Prospect performance evaluation: Making a case for a non-asymptotic UMPU test, Journal of Financial Econometrics, 10(4), 703-732.

Bai, Z.D., Li, H., Liu, H.X., Wong, W.K., 2011, Test statistics for prospect and Markowitz stochastic dominances with applications, Econometrics Journal, 122, 1-26.

Bai, Z.D., Li, H., McAleer, M., Wong, W.K., 2015, Stochastic dominance statistics for risk averters and risk seekers: An analysis of stock preferences for USA and China, Quantitative Finance, 15(5), 889-900.

Bai, Z.D., Li, H., McAleer, M., Wong, W.K., 2016, Spectrally-corrected estimation for highdimensional Markowitz mean-variance optimization, Tinbergen Institute Discussion Paper, TI 2016-025/III. 
Bai, Z.D., Li, H., Wong, W.K., Zhang, B.Z., 2011, Multivariate causality tests with simulation and application, Statistics and Probability Letters, 81(8), 1063-1071.

Bai, Z.D., Liu, H.X., Wong, W.K., 2009a, Enhancement of the applicability of Markowitz's portfolio optimization by utilizing random matrix theory, Mathematical Finance, 19(4), 639-667.

Bai, Z.D., Liu, H.X., Wong, W.K., 2009b, On the Markowitz mean-variance analysis of selffinancing portfolios, Risk and Decision Analysis, 1(1), 35-42.

Bai, Z.D., Phoon, K.F., Wang, K.Y., Wong, W.K., 2013, The performance of commodity trading advisors: A mean-variance-ratio test approach, North American Journal of Economics and Finance, 25, 188-201.

Bai, Z.D., Valenzuela, M.R., Wong, W.K., Zhu, Z.Z., 2016, New tests for poorness, richness, and middle class welfare: SD analysis for different types of social welfare functions, Social Science Research Network Working Paper 2742024.

Bai, Z.D., Wang, K.Y., Wong, W.K., 2011, Mean-variance ratio test, a complement to coefficient of variation test and Sharpe ratio test, Statistics and Probability Letters, 81(8), 10781085.

Bai, Z.D., Wong, W.K., Zhang, B.Z., 2010, Multivariate linear and non-linear causality tests, Mathematics and Computers in Simulation, 81, 5-17.

Barberis, N., Shleifer, A., Vishny, K., 1998, A model of investor sentiment, Journal of Financial Economics, 49, 307-343.

Batai, A., Chu, A.M.Y., Lv, Z., Wong, W.K., 2017, China’s impact on Mongolian exchange rate, Journal of Management Information and Decision Sciences, forthcoming.

Bawa, V.S., Bodurtha Jr. J.N., Rao, M.R., Suri, H.L., 1985, On determination of stochastic dominance optimal sets, Journal of Finance, 40(2), 417-431.

Bawa, V.S., Lindenberg, E.B., Rafsky, L.C., 1979, An efficient algorithm to determine stochastic dominance admissible sets, Management Science, 25(7), 609-622.

Bernoulli, D. (1738; translated by Dr. Louise Sommer 1954, Exposition of a new theory on the measurement of risk, Econometrica, 22(1), 22-36.

Bian, G., Dickey, J.M., 1996, Properties of multivariate Cauchy and poly-Cauchy distributions with Bayesian g-prior applications., in Bayesian Analysis in Statistics and Econometrics: Essays in Honor of Arnold Zellner, edited by Berry, D.A., Chaloner, K.M., Geweke, J.K., Wiley, New York, 299-310.

Bian, G., McAleer, M., Wong, W.K., 2011, A trinomial test for paired data when there are many ties, Mathematics and Computers in Simulation, 81(6), 1153-1160. 
Bian, G., McAleer, M., Wong, W.K., 2013, Robust estimation and forecasting of the capital asset pricing model, Annals of Financial Economics, 1350007, DOI: 10.1142/S2010495213500073.

Bian, G., Wong, W.K., (1997), An alternative approach to estimate regression coefficients, Journal of Applied Statistical Science, 6(1), 21-44.

Bouri, E., Gupta, R., Wong, W.K., Zhu, Z.Z., 2017, Is wine a good choice for investment?, Pacific-Basin Finance Journal, first revision.

Broll, U., Egozcue, M., Wong, W.K., Zitikis, R., 2010, Prospect theory, indifference curves, and hedging risks, Applied Mathematics Research Express, 2010(2), 142-153.

Broll, U., Guo, X., Welzel, P., Wong, W.K., 2015, The banking firm and risk taking in a twomoment decision model, Economic Modelling, 50, 275-280.

Broll, U., Wahl, J.E., Wong, W.K., 2006, Elasticity of risk aversion and international trade, Economics Letters, 91(1), 126-130.

Broll, U., Wong, W.K., Wu, M., 2011, Banking firm, risk of investment and derivatives, Technology and Investment, 2, 222-227.

Chan, C.-Y., de Peretti, C., Qiao, Z. Wong, W.K., 2012, Empirical test of the efficiency of the UK covered warrants market: Stochastic dominance and likelihood ratio test approach, Journal of Empirical Finance, 19(1), 162-174.

Chan, R.H., Clark, E., Wong, W.K., 2016, On the third order stochastic dominance for riskaverse and risk-seeking investors with analysis of their traditional and internet stocks, MPRA Paper No. 75002, University Library of Munich, Germany.

Chan, R.H., Lee, S.T.H., Wong, W.K., 2014, Technical analysis and financial asset forecasting: From simple tools to advanced techniques, World Scientific.

Chang, C.-L., McAleer, M., Wong, W.K., 2016a, Behavioural, financial, and health \& medical economics: A connection, Journal of Health \& Medical Economics, 2(1:1), 1-4.

Chang, C.-L., McAleer, M., Wong, W.K., 2016b, Informatics, data mining, econometrics and financial economics: A connection, Journal of Informatics and Data Mining, 1(1:7), 1-5.

Chang, C.-L., McAleer, M., Wong, W.K., 2016c, Management science, economics and finance: A connection, International Journal of Economics and Management Sciences, 5(4), 119.

Chang, C.-L., McAleer, M., Wong, W.K., 2017a, Management information, decision sciences, and financial economics: A connection, Journal of Management Information and Decision Sciences, 20th Anniversary special issue, 20(A), 2017, 3:1-21. 
Chang, C.-L., McAleer, M., Wong, W.K., 2017b, Big data, computational science, economics, finance and management: Connections, Journal of Risk and Financial Management, forthcoming.

Chang, W.C., Wong, W.K., Koh, B.K., 2003, Chinese values in Singapore: Traditional and modern, Asian Journal of Social Psychology, 6, 5-29.

Chang, W.C., Wong, W.K., Teo, G., Fam, A., 1997, The motivation to achieve in Singapore: In search of a core construct, Personality and Individual Differences, 23(5), 885-895.

Chang, W.C., Wong, W.K., Teo, G., 2000, The socially oriented and individually oriented achievement motivation of Singaporean Chinese students, Journal of Psychology in Chinese Societies, 1(2), 39-64.

Chiang, T.C., Qiao, Z., Wong, W.K., 2009, New evidence on the relation between return volatility and trading volume, Journal of Forecasting, 29(5), 502-515.

Chong, T.T.L., Cao, B.Q., Wong, W.K., 2017, A principal component approach to measuring investor sentiment in Hong Kong, Journal of Management Sciences, 4(2), 237-247.

Chow, S.C, Cunado, J., Gupta, R., Wong, W.K., 2017, Causal relationships between economic policy uncertainty and housing market returns in China and India: Evidence from linear and nonlinear panel and time series models, Studies in Nonlinear Dynamics and Econometrics, forthcoming.

Chow, S.C., Lui, F.T., Valenzuela, M.R., Wong, W.K., 2015, Tests for richness and poorness: A stochastic dominance analysis of income distributions in Hong Kong, presented at the Sixth Meeting of the Society for the Study of Economic Inequality (ECINEQ), Université du Luxembourg.

Clark, E.A., Qiao, Z., Wong, W.K., 2016, Theories of risk: testing investor behaviour on the Taiwan stock and stock index futures markets, Economic Inquiry, 54(2), 907-924.

Currim, I.S., R.K. Sarin., 1989, Prospect versus utility, Management Science, 35, 22-41.

Davidson, R., Duclos, J.Y., 2000, Statistical inference for stochastic dominance and for the measurement of poverty and inequality, Econometrica, 68, 1435-1464.

De Miguel, V., Garlappi, L., Uppal, R., 2009, Optimal versus naive diversification: How inefficient is the 1/N portfolio strategy?, Review of Financial Studies, 22(5), 1915-1953.

Egozcue, M., Fuentes García, F., Wong, W.K., 2009, On some covariance inequalities for monotonic and non-monotonic functions, Journal of Inequalities in Pure and Applied Mathematics, 10(3), 75:1-7. 
Egozcue, M., Fuentes García, F., Wong, W.K., Zitikis, R., 2010, Grüss-type bounds for the covariance of transformed random variables, Journal of Inequalities and Applications, 2010, Article ID 619423, 1-10.

Egozcue, M., Fuentes García, F., Wong, W.K., Zitikis, R., 2011, Do investors like to diversify? A study of Markowitz preferences, European Journal of Operational Research, 215(1), 188-193.

Egozcue, M., Fuentes García, F., Wong, W.K., Zitikis, R., 2011a, Grüss-type bounds for covariances and the notion of quadrant dependence in expectation, Central European Journal of Mathematics, 9(6), 1288-1297.

Egozcue, M., Fuentes García, F., Wong, W.K., Zitikis, R., 2011b, The covariance sign of transformed random variables with applications to economics and finance, IMA Journal of Management Mathematics, 22(3), 291-300.

Egozcue, M., Fuentes García, F., Wong, W.K., Zitikis, R., 2012, The smallest upper bound for the $\mathrm{p}^{\text {th }}$ absolute central moment of a class of random variables, The Mathematical Scientist 37, 17.

Egozcue, M., Fuentes García, F., Wong, W.K., Zitikis, R., 2012a, Integration-segregation decisions under general value functions: 'Create your own bundle - choose 1, 2, or all 3!, IMA Journal of Management Mathematics, 1-16, doi:10.1093/imaman/dps024

Egozcue, M., Fuentes García, F., Wong, W.K., Zitikis, R., 2013, Convex combinations of quadrant dependent copulas, Applied Mathematics Letters, 26(2), 249-251.

Egozcue, M., Guo, X., Wong, W.K., 2015, Optimal output for the regret-averse competitive firm under price uncertainty, Eurasian Economic Review, 5(2), 279-295.

Egozcue, M., Wong, W.K., 2010, Gains from diversification: A majorization and stochastic dominance approach, European Journal of Operational Research, 200(3), 893-900.

Egozcue, M., Wong, W.K., 2010a, Segregation and integration: A study of the behaviors of investors with extended value functions, Journal of Applied Mathematics and Decision Sciences, 2010, Article ID 302895, 1-8.

Fabozzi, F.J., Fung, C.Y., Lam, K., Wong, W.K., 2013, Market overreaction and underreaction: Tests of the directional and magnitude effects, Applied Financial Economics, 23(18), 1469-1482.

Falk, H., H. Levy, 1989, Market reaction to quarterly earnings' announcements: A stochastic dominance based test of market efficiency, Management Science, 35(4), 425-446.

Farinelli, S., Tibiletti, L., 2008, Sharpe thinking in asset ranking with one-sided measures. European Journal of Operational Research, 185(3), 1542-1547. 
Fishburn, P.C., 1974, Convex stochastic dominance with continuous distribution functions, Journal of Economic Theory, 7, 143-58.

Fong, W.M., H.H. Lean, W.K. Wong, 2008, Stochastic dominance and behavior towards risk: The market for internet stocks, Journal of Economic Behavior and Organization, 68(1), 194208.

Fong, W.M., Wong, W.K., 2006, The Modified Mixture of Distributions Model: A Revisit, Annals of Finance, 2(2), 167-178.

Fong, W.M., Wong, W.K., Lean, H.H., 2005, International momentum strategies: A stochastic dominance approach, Journal of Financial Markets, 8, 89-109.

Foo, S.Y., Wong, W.K., Chong, T.T.L., 2008, Are the Asian equity markets more interdependent after the financial crisis?, Economics Bulletin, 6(16), 1-7.

Fry-McKibbin, R., Hsiao, C.Y-L., 2015, Extremal dependence tests for contagion, Econometric Reviews, DOI: 10.1080/07474938.2015.1122270.

Fry, R., Martin, V.L., Tang, C., 2010, A new class of tests of contagion with application, Journal of Business \& Economic Statistics, 28, 423-437.

Fung, E.S., Lam, K., Siu, T.K., Wong, W.K., 2011, A new pseudo Bayesian model for financial crisis, Journal of Risk and Financial Management, 4, 42-72.

Gasbarro, D., Wong, W.K., Zumwalt, J.K., 2007, Stochastic dominance analysis of iShares, European Journal of Finance, 13, 89-101.

Gasbarro, D., Wong, W.K., Zumwalt, J.K., 2012, Stochastic dominance and behavior towards risk: The market for iShares, Annals of Financial Economics, 7(1), 1250005-1-20.

Gotoh, J.-Y., H. Konno, 2000, Third-degree stochastic dominance and mean-risk analysis, Management Science, 46, 289-301.

Gordon, M.J., Shapiro, E., 1956, Capital equipment analysis: the required rate of profit, Management Science, X, 102-110.

Guo, X., Egozcue, M., Wong, W.K., 2017, Optimal production decision with disappointment aversion under uncertainty, Social Science Research Network, Working Paper Series 3036742.

Guo, X., Jiang, X.J., Wong, W.K., 2017,, Stochastic dominance and Omega ratio: Measures to examine market efficiency, Arbitrage Opportunity, and Anomaly, Economies 5, 4:38. 
Guo, X., Lien, D., Wong, W.K., 2016, Good approximation of exponential utility function for optimal futures hedging, Journal of Mathematical Finance, 6, 457-463.

Guo, X., McAleer, M., Wong, W.K., Zhu, L.X., 2017, A Bayesian approach to excess volatility, short-term underreaction and long-term overreaction during financial crises, North American Journal of Economics and Finance, 42, 346-358.

Guo, X., Post, T. Wong, W.K., Zhu, L.X., 2014, Moment conditions for almost stochastic dominance, Economics Letters, 124(2), 163-167.

Xu Guo, X., Qiao, Z., Wong, W.K., 2017, Why investors buy insurance and try their luck with lotteries as well?, Social Science Research Network, Working Paper Series 2980007.

Guo, X., Wagener, A., Wong, W.K., Zhu, L.X., 2017, The two-moment decision model with additive risks, Risk Management, forthcoming.

Guo, X., Wong, W.K., 2016, Multivariate stochastic dominance for risk averters and risk seekers, RAIRO - Operations Research, 50(3), 575-586.

Guo, X., Wong, W.K., Xu, Q.F., Zhu, L.X., 2015, Production and hedging decisions under regret aversion, Economic Modelling, 51, 153-158.

Guo, X., Wong, W.K., Zhu, L.X., 2016, Almost stochastic dominance for risk averters and risk seekers, forthcoming in Finance Research Letters.

Guo, X., Zhu, X.H., Wong, W.K., Zhu, L.X., 2013, A note on almost stochastic dominance, Economics Letters, 121(2), 252-256.

Hammond, J.S., 1974, Simplifying the choice between uncertain prospects where preference is nonlinear, Management Science, 20(7), 1047-1072.

Hanoch, G., H. Levy, 1969, The efficiency analysis of choices involving risk, Review of Economic Studies, 36, 335-346.

Hiemstra, C., Jones, J.D., 1994, Testing for linear and nonlinear Granger causality in the stock price-volume relation, Journal of Finance, 49(5), 1639-1664.

Hoang, T.H.V., Lean, H.H., Wong, W.K., 2015, Is gold good for portfolio diversification? A stochastic dominance analysis of the Paris stock exchange, International Review of Financial Analysis, 42, 98-108.

Hoang, V.T.H., Wong, W.K., Zhu, Z.Z., 2015, Is gold different for risk-averse and risk-seeking investors? An empirical analysis of the Shanghai Gold Exchange, Economic Modelling, 50, 200211.

Homm, U. and Pigorsch, C., 2012, Beyond the Sharpe ratio: An application of the AumannSerrano index to performance measurement, Journal of Banking \& Finance, 36, 2274-2284 
Hui, Y.C., Wong, W.K., Bai, Z.D., Zhu, Z.Z., 2017, A new nonlinearity test to circumvent the limitation of Volterra expansion with application, Journal of the Korean Statistical Society, 46(3), 365-374.

Kahneman, D., Tversky, A., 1979, Prospect theory of decisions under risk, Econometrica, 47(2), 263-291.

Kung, J.J., Wong, W.K., 2009a, Profitability of technical analysis in Singapore stock market: Before and after the Asian financial crisis, Journal of Economic Integration, 24(1), 133-150.

Kung, J.J., Wong, W.K., 2009b, Efficiency of the Taiwan stock market, Japanese Economic Review, 60(3), 389-394.

Kung, J.J., Wong, W.K., Wu, E.C., 2013, Stochastic control for asset management, Journal of Mathematical Finance, 3(1), 59-69, doi: 10.4236/jmf.2013.31005.

Kuosmanen, T., 2004, Efficient diversification according to stochastic dominance criteria, Management Science, 50(10), 1390-1406.

Korkie, B., H.J. Turtle, 2002, A mean-variance analysis of self-financing portfolio, Management Science, 48(3), 427-444.

Lam, K., Liu, T.S., Wong, W.K., 2010, A pseudo-Bayesian model in financial decision making with implications to market volatility, under- and overreaction, European Journal of Operational Research, 203(1), 166-175.

Lam, K., Liu, T.S., Wong, W.K., 2012, A new pseudo Bayesian model with implications to financial anomalies and investors' behaviors, Journal of Behavioral Finance, 13(2), 93-107.

Lam, K., Wong, C.M., Wong, W.K., 2006, New variance ratio tests to identify random walk from the general mean reversion model, Journal of Applied Mathematics and Decision Sciences/Advances in Decision Sciences, 2006, 1-21.

Lam, V.W.S., Chong, T.T.L., Wong, W.K., 2007, Profitability of intraday and interday momentum strategies, Applied Economics Letters, 14, 1103-1108.

Leshno, M., Levy, H., 2002, Preferred by “all” and preferred by “most” decision makers: Almost stochastic dominance, Management Science, 48(8), 1074-1085.

Lean, H.H., McAleer, M., Wong, W.K., 2010, Market efficiency of oil spot and futures: A meanvariance and stochastic dominance approach, Energy Economics, 32, 979-986.

Lean, H.H., McAleer, M., Wong, W.K., 2015, Preferences of risk-averse and risk-seeking investors for oil spot and futures before, during and after the global financial crisis, International Review of Economics and Finance, 40, 204-216. 
Lean, H.H., Phoon, K.F., Wong, W.K., 2012, Stochastic dominance analysis of CTA funds, Review of Quantitative Finance and Accounting, 40(1), 155-170.

Lean, H.H., Smyth, R. Wong, W.K., 2007, Revisiting calendar anomalies in Asian stock markets using a stochastic dominance approach, Journal of Multinational Financial Management, 17(2), $125-141$.

Leung, P.L., Ng, H.Y., Wong, W.K., 2012, An improved estimation to make Markowitz's portfolio optimization theory users friendly and estimation accurate with application on the US stock market investment, European Journal of Operational Research, 222(1), 85-95.

Leung, P.L., Wong, W.K., 2008, On testing the equality of the multiple Sharpe ratios, with application on the evaluation of Ishares, Journal of Risk, 10(3), 1-16.

Levy, H., 2015, Stochastic dominance: Investment decision making under uncertainty, Third Edition, Springer, New York,

Levy, H., M. Levy, 2004, Prospect theory and mean-variance analysis, Review of Financial Studies, 17(4), 1015-1041.

Levy, M., H. Levy, 2002, Prospect theory: Much ado about nothing?, Management Science, 48(10), 1334-1349.

Li, C.K., W.K. Wong, 1999, Extension of stochastic dominance theory to random variables, RAIRO - Operations Research, 33(4), 509-524.

Li, Z.G., Li, X.G., Hui, Y.C., Wong, W.K., 2017, Maslow portfolio selection for individuals with low financial sustainability, working paper.

Liao, Z.Q., Shi, X.P., Wong, W.K., 2012, Consumer perceptions of the smartcard in retailing: An empirical study, Journal of International Consumer Marketing, 24(4), 252-262.

Liao, Z.Q., Shi, X.P., Wong, W.K., 2014, Key determinants of sustainable smartcard payment, Journal of Retailing and Consumer Services, 21(3), 306-313.

Liao, Z.Q., Wong, W.K., 2008, The determinants of customer interactions with internet-enabled e-banking services, Journal of the Operational Research Society, 59(9), 1201-1210.

Liew, V.K.S., Qiao, Z., Wong, W.K., 2010, Linearity and stationarity of G7 government bond returns, Economics Bulletin, 30(4), 1-13.

Lozza, S.,O., Wong, W.K., Fabozzi, F.J., Egozcue, M., 2017, Diversification versus optimal: Is there really a diversification puzzle? Applied Economics, first revision.

Ma, C., Wong, W.K., 2010, Stochastic dominance and risk measure: A decision-theoretic foundation for VaR and C-VaR, European Journal of Operational Research, 207(2), 927-935. 
Manzur, M., Wong, W.K., Chau, I.C., 1999, Measuring international competitiveness: Experience from East Asia, Applied Economics, 31(11), 1383-1391.

Markowitz, H.M., 1952a, The utility of wealth, Journal of Political Economy, 60, 151-156.

Markowitz, H.M., 1952b, Portfolio selection, Journal of Finance, 7, 77-91.

Maslow, A.H., 1943, A theory of human motivation, Psychological Review 50, 370-396.

Matsumura, E.M., Tsui, K.W., Wong, W.K. (1990), An extended multinomial-Dirichlet model for error bounds for dollar-unit sampling, Contemporary Accounting Research, 6(2-I), 485500.

McAleer, M., Suen, J., Wong, W.K., 2016, Profiteering from the dot-com bubble, subprime crisis and Asian financial crisis, Japanese Economic Review, 67(3), 257-279.

Meyer, J., 1977, Second degree stochastic dominance with respect to a function, International Economic Review, 18, 476-487.

Meyer, J., 1987, Two-moment decision models and expected utility maximization, American Economic Review, 77, 421-430.

Mroua, M., Abid, F., Wong, W.K., 2017, Optimal diversification, stochastic dominance, and sampling error, American Journal of Business, 32(1), 58-79.

Moslehpour, M., Pham, V.K., Wong, W.K., Bilgiçli, I., 2017, Online purchase intention of Taiwanese consumers: Sustainable effects of personality traits and technology perception attributes, Sustainability, forthcoming.

Moslehpour, M., Wong, W.K., Aulia, C.K., Pham, V.K., 2017, Repurchase intention of Korean beauty products among Taiwanese consumers, Asia Pacific Journal of Marketing and Logistics, 29(3), 569-588.

Moslehpour, M., Wong, W.K., Lin, Y.H., Huyen, N.T.L., 2017, Mediating role of customer satisfaction toward Vietjet Air's purchase intention in Vietnam, Eurasian Business Review, forthcoming.

Ng, P., Wong, W.K., Xiao, Z.J., 2017, Stochastic dominance via quantile regression, European Journal of Operational Research, 261(2), 666-678.

Niu, C.Z., Guo, X., McAleer, M., Wong, W.K., 2017, Theory and application of an economic performance measure of risk, International Review of Economics \& Finance, forthcoming.

Niu, C.Z., Wong, W.K., Zhu, L.X., 2016, First stochastic dominance and risk measurement, MPRA Paper 75027, University Library of Munich, Germany. 
Niu, C.Z., Wong, W.K., Xu, Q.F., 2017, Kappa ratios and (higher-order) stochastic dominance, Risk Management, 19(3), 245-253.

Phang, S.Y., Wong, W.K., 1997, Government policies and private housing prices in Singapore, Urban Studies, 34(11), 1819-1829.

Phang, S.Y., Wong, W.K., Chia, N.C., 1996, Singapore's experience with car quotas: Issues and policy processes, Transport Policy, 3, 145-153.

Qiao, Z., Clark, E., Wong, W.K., 2012, Investors’ preference towards risk: Evidence from the Taiwan stock and stock index futures markets, Accounting Finance, 54(1), 251-274.

Qiao, Z., Chiang, T.C., Wong, W.K., 2008, Long-run equilibrium, short-term adjustment, and spillover effects across Chinese segmented stock markets, Journal of International Financial Markets, Institutions \& Money, 18, 425-437.

Qiao, Z., Li, Y.M., Wong, W.K., 2008, Policy change and lead-lag relations among China's segmented stock markets, Journal of Multinational Financial Management, 18, 276-289.

Qiao, Z., Li, Y.M., Wong, W.K., 2011, Regime-dependent relationships among the stock markets of the US, Australia, and New Zealand: A Markov-switching VAR approach, Applied Financial Economics, 21(24), 1831-1841.

Qiao, Z., Liew, V.K.S., Wong, W.K., 2007, Does the US IT stock market dominate other IT stock markets: Evidence from multivariate GARCH model, Economics Bulletin, 6(27), 1-7.

Qiao, Z., McAleer, M., Wong, W.K., 2009, Linear and nonlinear causality between changes in consumption and consumer attitudes, Economics Letters, 102(3), 161-164.

Qiao, Z., Qiao, W.W., Wong, W.K., 2010, Examining the day-of-the-week effects in Chinese stock markets: New evidence from a stochastic dominance approach, Global Economic Review, 39(3), 225-246.

Qiao, Z., Smyth, R., Wong, W.K., 2008, Volatility switching and regime interdependence between information technology stocks 1995-2005, Global Finance Journal, 19, 139-156.

Qiao, Z., Wong, W.K., 2015, Which is a better investment choice in the Hong Kong residential property market: A big or small property?, Applied Economics, 47(16), 1670-1685.

Qiao, Z., Wong, W.K., Fung, J.K.W., 2013, Stochastic dominance relationships between stock and stock index futures markets: International evidence, Economic Modelling, 33, 552-559.

Quirk J.P., Saposnik R., 1962, Admissibility and measurable utility functions, Review of Economic Studies, 29, 140-146.

Sethi, D., Wong, W.K., Acharya, D., 2017, Can a disinflationary policy have a differential 
impact on sectoral output? A look at sacrifice ratios in OECD and non-OECD countries, Margin: Journal of Applied Economic Research, forthcoming.

Sharpe, W.F., 1966, Mutual Fund Performance, Journal of Business, 39(1), 119-138.

Sriboonchitta, S., Wong, W.K., Dhompongsa, S., Nguyen, H.T., 2009, Stochastic dominance and applications to finance, risk and economics, Chapman and Hall/CRC, Taylor and Francis, Boca Raton, Florida, USA.

Stoyan, D., 1983, Comparison Methods for Queues and Other Stochastic Models, New York, Wiley.

Tobin, J., 1958, Liquidity preference and behavior towards risk, Review of Economic Studies, 25, 65-86.

Thaler, R.H., Johnson, E.J., 1990, Gambling with the house money and trying to break even: The effects of prior outcomes on risky choice, Management Science, 36, 643-660.

Thompson, H.E., 1985, The magnitude and reliability of equity capital cost estimates: A statistical approach, Managerial and Decision Economics, 6, 132-140.

Thompson, H.E., Wong, W.K., 1991, On the unavoidability of "unscientific" judgement in estimating the cost of capital, Managerial and Decision Economics, 12, 27-42.

Thompson, H.E., Wong, W.K., 1996, Revisiting 'dividend yield plus growth' and its applicability, Engineering Economist, 41(2), 123-147.

Tiku, M.L., Wong, W.K., 1998, Testing for unit root in AR(1) model using three and four moment approximations, Communications in Statistics: Simulation and Computation, 27(1), 185-198.

Tiku, M.L., Wong W.K., Bian, G., 1999, Time series models with asymmetric innovations, Communications in Statistics: Theory and Methods, 28(6), 1331-1360.

Tiku, M.L., Wong W.K., Bian, G., 1999a, Estimating parameters in autoregressive models in non-normal situations: Symmetric innovations, Communications in Statistics: Theory and Methods, 28(2), 315-341.

Tiku, M.L., Wong, W.K., Vaughan, D.C., Bian, G., 2000, Time series models in non-normal situations: Symmetric innovations, Journal of Time Series Analysis, 21, 571-96.

Tsang, C.K., Wong, W.K., Horowitz, I., 2016, Arbitrage opportunities, efficiency, and the role of risk preferences in the Hong Kong property market, Studies in Economics and Finance, 33(4), 735-754. 
Tsui, K.W., Matsumura, E.M., Tsui, K.L., 1985, Multinomial-Dirichlet bounds for dollar-unit sampling in auditing, Accounting Review, 60 (1), 76-96.

Tzeng, L.Y., Huang, R.J., Shih, P.-T., 2013, Revisiting almost second-degree stochastic dominance, Management Science, 59, 1250-1254.

Tsetlin, I., Winkler, R.L., Huang, R.J., Tzeng, L.Y., 2015, Generalized almost stochastic dominance, Operations Research, 62(2), 363-377.

Vieito, J.P., Wong, W.K., Zhu, Z.Z., 2015, Could the global financial crisis improve the performance of the G7 stocks markets?, Applied Economics, 48(12) 1066-1080.

Wan, H.J., Wong, W.K., 2001, Contagion or inductance? Crisis 1997 reconsidered, Japanese Economic Review, 52(4), 372-380.

Wong, W.K., 2006, Stochastic dominance theory for location-scale family, Journal of Applied Mathematics and Decision Sciences, 2006, 1-10.

Wong, W.K., 2007, Stochastic dominance and mean-variance measures of profit and loss for business planning and investment, European Journal of Operational Research, 182(2), 829-843.

Wong, W.K., Agarwal, A., Du, J., 2004, Financial integration for India stock market, a fractional cointegration approach, Finance India, 18(4), 1581-1604.

Wong, W.K., Bian, G., 2000, Robust estimation in capital asset pricing estimation, Journal of Applied Mathematics \& Decision Sciences, 4(1), 65-82.

Wong, W.K., Bian, G., 2005, Estimating parameters in autoregressive models with asymmetric innovations, Statistics and Probability Letters, 71(1), 61-70.

Wong, W.K., Chan, R., 2004, On the estimation of cost of capital and its reliability, Quantitative Finance, 4(3), 365-372.

Wong, W.K., Chan, R., 2008, Markowitz and prospect stochastic dominances, Annals of Finance, 4(1), 105-129.

Wong, W.K., Chew, B.K., Sikorski, D., 2001, Can P/E ratio and bond yield be used to beat stock markets?. Multinational Finance Journal, 5(1), 59-86.

Wong, W.K., Chow, S.C., Hon, T.Y., Woo, K.Y., 2017, Empirical study on conservative and representative heuristics of Hong Kong small investors adopting momentum and contrarian trading strategies, International Journal of Revenue Management, forthcoming.

Wong, W.K., Du, J., Chong, T.T.L., 2005, Do the technical indicators reward chartists in Greater China stock exchanges?m Review of Applied Economics, 1(2), 183-205. 
Wong, W.K., Khan, H., Du, J., 2006, Money, interest rate, and stock prices: New evidence from Singapore and USA, Singapore Economic Review, 51(1), 31-52.

Wong, W.K., C.K. Li., 1999, A note on convex stochastic dominance theory, Economics Letters, 62, 293-300.

Wong, W.K., Ma, C., 2008, Preferences over location-scale family, Economic Theory, 37(1), 119-146.

Wong, W.K., Manzur, M., Chew, B.K., 2003, How rewarding is technical analysis? Evidence from Singapore stock market, Applied Financial Economics, 13(7), 543-551.

Wong, W.K., Miller, R.B., 1990, Repeated time series analysis of ARIMA-noise models, Journal of Business and Economic Statistics, 8(2), 243-250.

Wong, W.K, Miller, R.B., Shrestha, K., 2001, Maximum likelihood estimation of ARMA model with error processes for replicated observation, Journal of Applied Statistical Science, 10(4), 287-297.

Wong, W.K., Penm, J.H.W., Terrell, R.D., Lim, K.Y.C., 2004, The relationship between stock markets of major developed countries and Asian emerging markets, Advances in Decision Sciences, 8(4), 201-218.

Wong, W.K., Phoon, K.F., Lean, H.H., 2008, Stochastic dominance analysis of Asian hedge funds, Pacific-Basin Finance Journal, 16(3), 204-223.

Wong, W.K., Thompson, H.E., Wei, S., Chow, Y.F., 2006, Do winners perform better than losers? A stochastic dominance approach, Advances in Quantitative Analysis of Finance and Accounting, 4, 219-254.

Wong, W.K., Wright, J.A., Yam, S.C.P., Yung, S.P., 2012, A mixed Sharpe ratio, Risk and Decision Analysis, 3(1-2), 37-65.

Xiao, J.L., Brooks, R.D. Wong, W.K., 2009, GARCH and volume effects in the Australian stock markets, Annals of Financial Economics, 5, 79-105.

Xu, R.H., Wong, W.K., Chen, G.R., Huang, S., 2017, Topological characteristics of the Hong Kong stock market: A test-based P-threshold approach to understanding network complexity, Scientific Reports, 7, 41379, doi:10.1038/srep41379.

Zheng, Y., Chen, H., Wong, W.K., 2009, China's stock market integration with a leading power and a close neighbor, Journal of Risk and Financial Management, 2, 38-74. 